\title{
Carbolithiation for the Generation of Cyclooctadienyl Anions and Tandem Electrocyclization/Alkylation to Functionalized cis-Bicyclo[3.3.0]octenes
}

David R. Williams* and Jonathan T. Reeves

Department of Chemistry, Indiana University, Bloomington, Indiana 47405-7102

\section{Supporting Information}

General. All reactions were performed under an atmosphere of argon and all glassware was flame-dried prior to use. THF and $\mathrm{Et}_{2} \mathrm{O}$ were dried by distillation over $\mathrm{Na}$ /benzophenone. Unless otherwise stated, solvents or reagents were used without further purification. NMR spectra were recorded at $400 \mathrm{MHz} / 100 \mathrm{MHz}\left({ }^{1} \mathrm{H} /{ }^{13} \mathrm{C} \mathrm{NMR}\right)$ in $\mathrm{CDCl}_{3}$.

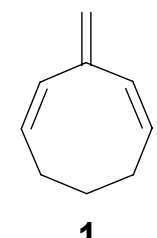

3-Methylene-1,4-cyclooctadiene (1). A suspension of methyltriphenylphosphonium bromide $(10.54 \mathrm{~g}, 29.50 \mathrm{mmol})$ in $\mathrm{Et}_{2} \mathrm{O}(300 \mathrm{~mL})$ was treated dropwise at $-78{ }^{\circ} \mathrm{C}$ with $n$ $\mathrm{BuLi}(11.8 \mathrm{~mL}, 29.5 \mathrm{mmol}, 2.5 \mathrm{M} /$ hexanes $)$. The reaction mixture was stirred at $0{ }^{\circ} \mathrm{C}$ for $1.5 \mathrm{~h}$, recooled to $-78^{\circ} \mathrm{C}$ and treated dropwise with a solution of 2,7-cyclooctadienone $(3.00 \mathrm{~g}, 24.6 \mathrm{mmol})$ in $\mathrm{Et}_{2} \mathrm{O}(10 \mathrm{~mL})$. The reaction mixture was stirred at $-78^{\circ} \mathrm{C}$ for $1 \mathrm{~h}$, quenched with saturated aqueous $\mathrm{NaHCO}_{3}$ solution, and extracted with $\mathrm{Et}_{2} \mathrm{O}$. The combined organic extracts were dried $\left(\mathrm{Na}_{2} \mathrm{SO}_{4}\right)$, filtered and concentrated (bath temperature $=0-5{ }^{\circ} \mathrm{C}$ ). The resultant crude product was diluted with pentane and filtered through a short pad of basic activated $\mathrm{Al}_{2} \mathrm{O}_{3}$, rinsing with pentane, and the combined pentane filtrate was concentrated (bath temperature $\left.=0-5{ }^{\circ} \mathrm{C}\right)$ to provide pure $1(1.89 \mathrm{~g}$, $64 \%$ ) as a colorless liquid. Compound 1 slowly decomposes by polymerization and is best stored at $-20{ }^{\circ} \mathrm{C}$ as a solution $(\sim 0.2 \mathrm{M})$ in pentane and filtered through basic activated $\mathrm{Al}_{2} \mathrm{O}_{3}$ immediately prior to use. IR (neat) $3019,2933,2868,1569,1458,886$, $772 \mathrm{~cm}^{-1}$; ${ }^{1} \mathrm{H}$ NMR $\delta 6.31(\mathrm{~d}, 2 \mathrm{H}, J=11.6 \mathrm{~Hz}), 5.42(\mathrm{dt}, 2 \mathrm{H}, J=8.4,11.6 \mathrm{~Hz}), 5.01(\mathrm{~s}$, $2 \mathrm{H}), 2.32(\mathrm{dt}, 4 \mathrm{H}, J=6.4,8.4 \mathrm{~Hz}), 1.43$ (p, $2 \mathrm{H}, J=6.4 \mathrm{~Hz}) ;{ }^{13} \mathrm{C}$ NMR $\delta 143.5,133.8$, 129.1, 119.2, 24.6, 22.4; MS (EI) $\mathrm{m} / z$ (rel. intensity) $120\left(\mathrm{M}^{+}, 43\right), 105$ (45) 91 (100); HRMS (EI) calcd for $\mathrm{C}_{9} \mathrm{H}_{12}$ : 120.0939 , found: 120.0934 .

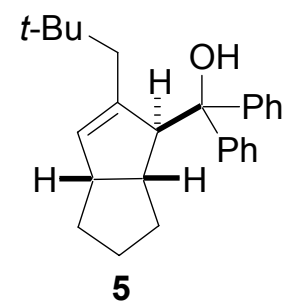


[2-(2,2-Dimethylpropyl)-1,3a,4,5,6,6a-hexahydropentalen-1-yl] diphenylmethanol (5). A solution of $1(222 \mathrm{mg}, 1.85 \mathrm{mmol})$ in $\mathrm{Et}_{2} \mathrm{O}(8 \mathrm{~mL})$ was treated dropwise at $-78{ }^{\circ} \mathrm{C}$ with $t$-BuLi $(1.20 \mathrm{~mL}, 2.04 \mathrm{mmol}, 1.7 \mathrm{M} /$ pentane, 1.1 equiv). The reaction mixture was stirred at $-78{ }^{\circ} \mathrm{C}$ for $10 \mathrm{~min}$, then at $\mathrm{rt}$ for $1.5 \mathrm{~h}$. The reaction mixture was recooled to $78{ }^{\circ} \mathrm{C}$ and treated dropwise with a solution of benzophenone $(674 \mathrm{mg}, 3.70 \mathrm{mmol})$ in $\mathrm{Et}_{2} \mathrm{O}(2 \mathrm{~mL})$. After stirring at $-78{ }^{\circ} \mathrm{C}$ for $30 \mathrm{~min}$, the reaction mixture was warmed to $\mathrm{rt}$, quenched with saturated aqueous $\mathrm{NaHCO}_{3}$ solution, and extracted with $\mathrm{Et}_{2} \mathrm{O}$. The combined organic layers were dried $\left(\mathrm{MgSO}_{4}\right)$, filtered and concentrated. ${ }^{1} \mathrm{H}$ NMR of the crude product showed a $\mathrm{dr}>20: 1$. Column chromatography on $\mathrm{SiO}_{2}$ (hexanes/ $/ \mathrm{Et}_{2} \mathrm{O}$, 95:5) provided 5 (433 $\mathrm{mg}, 65 \%)$ as a colorless oil that solidified on standing. IR (neat) $3532,3028,2946,2857,1490,1445,1364,1165,746 \mathrm{~cm}^{-1} ;{ }^{1} \mathrm{H}$ NMR $\delta 7.65(\mathrm{~d}, 2 \mathrm{H}, J=$ $7.6 \mathrm{~Hz}), 7.55(\mathrm{~d}, 2 \mathrm{H}, J=7.6 \mathrm{~Hz}), 7.36(\mathrm{t}, 2 \mathrm{H}, J=7.6 \mathrm{~Hz}), 7.30(\mathrm{t}, 2 \mathrm{H}, J=7.6 \mathrm{~Hz}), 7.22$ (t, $2 \mathrm{H}, J=7.2 \mathrm{~Hz}), 7.17(\mathrm{t}, 2 \mathrm{H}, J=7.2 \mathrm{~Hz}), 5.57(\mathrm{~s}, 1 \mathrm{H}), 3.85(\mathrm{~s}, 1 \mathrm{H}), 3.18-3.09$ (br, 1 H), $2.68(\mathrm{~s}, 1 \mathrm{H}), 2.44(\mathrm{q}, 1 \mathrm{H}, J=7.6 \mathrm{~Hz}), 1.96-1.88(\mathrm{~m}, 1 \mathrm{H}), 1.77-1.68(\mathrm{~m}, 1 \mathrm{H}), 1.66-$ $1.50(\mathrm{~m}, 2 \mathrm{H}), 1.49-1.39(\mathrm{~m}, 3 \mathrm{H}), 0.76(\mathrm{~s}, 9 \mathrm{H}), 0.67(\mathrm{~d}, 1 \mathrm{H}, J=14.0 \mathrm{~Hz}) ;{ }^{13} \mathrm{C}$ NMR $\delta$ $148.1,140.09,140.05,128.3,128.2,126.3,126.0,125.7,79.7,65.5,48.7,46.5,42.0$, 35.0, 32.2, 29.8, 25.6; MS (EI) $\mathrm{m} / \mathrm{z}$ (rel. intensity) 183 (100), 105 (84); HRMS (EI) calcd for $\mathrm{C}_{26} \mathrm{H}_{31}$ (M-OH): 343.2426 , found: 343.2429 .

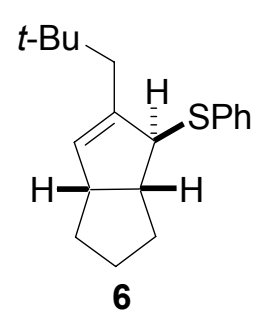

5-(2,2-Dimethylpropyl)-4-phenylsulfanyl-1,2,3,3a,4,6a-hexahydropentalene (6). A solution of $1(35.0 \mathrm{mg}, 0.292 \mathrm{mmol})$ in $\mathrm{Et}_{2} \mathrm{O}(2 \mathrm{~mL})$ was treated dropwise at $-78{ }^{\circ} \mathrm{C}$ with $t$-BuLi $(0.190 \mathrm{~mL}, 0.323 \mathrm{mmol}, 1.7 \mathrm{M} /$ pentane, 1.1 equiv). The reaction mixture was stirred at $-78{ }^{\circ} \mathrm{C}$ for $10 \mathrm{~min}$, then at $\mathrm{rt}$ for $1 \mathrm{~h}$. The reaction mixture was recooled to -78 ${ }^{\circ} \mathrm{C}$ and treated dropwise with a solution of PhSSPh $(67.0 \mathrm{mg}, 0.307 \mathrm{mmol})$ in $\mathrm{Et}_{2} \mathrm{O}(0.5$ $\mathrm{mL}$ ). The reaction mixture was allowed to warm to $\mathrm{rt}$ over $40 \mathrm{~min}$, quenched with saturated aqueous $\mathrm{NaHCO}_{3}$ solution, and extracted with $\mathrm{Et}_{2} \mathrm{O}$. The combined organic layers were dried $\left(\mathrm{MgSO}_{4}\right)$, filtered and concentrated. ${ }^{1} \mathrm{H}$ NMR of the crude product showed a $\mathrm{dr}=10: 1$. Column chromatography on $\mathrm{SiO}_{2}$ (hexanes) provided $6(59 \mathrm{mg}$, $71 \%$ ) as an inseparable 10:1 mixture of diastereomers and a colorless oil. IR (neat) 3025 , 2949, 2861, 1583, 1479, 1435, 1363, 1239, 1195, 1023, 736, $692 \mathrm{~cm}^{-1} ;{ }^{1} \mathrm{H}$ NMR $\delta$ 7.40$7.37(\mathrm{~m}, 2 \mathrm{H}), 7.33-7.20(\mathrm{~m}, 3 \mathrm{H}), 5.30(\mathrm{~s}, 1 \mathrm{H}), 3.89(\mathrm{~s}, 1 \mathrm{H}), 2.99-2.96(\mathrm{~m}, 1 \mathrm{H}), 2.82-$ $2.78(\mathrm{~m}, 1 \mathrm{H}), 2.28(\mathrm{~d}, 1 \mathrm{H}, J=13.6 \mathrm{~Hz}), 2.03(\mathrm{~d}, 1 \mathrm{H}, J=13.6 \mathrm{~Hz}), 1.83-1.77(\mathrm{~m}, 1 \mathrm{H})$, 1.60-1.43 (m, $5 \mathrm{H}), 0.94$ (s, $9 \mathrm{H}) ;{ }^{13} \mathrm{C}$ NMR $\delta 140.3,136.6,131.8,128.7,126.6,65.6$, $50.8,49.1,42.1,34.5,32.1,31.6,30.1,25.4$; MS (EI) $\mathrm{m} / z$ (rel. intensity) $286\left(\mathrm{M}^{+}, 11\right)$, 218 (34), 177 (86), 121(100); HRMS (EI) calcd for $\mathrm{C}_{19} \mathrm{H}_{26} \mathrm{~S}$ : 286.1755, found: 286.1749 . 


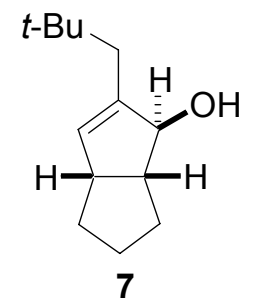

2-(2,2-Dimethylpropyl)-1,3a,4,5,6,6a-hexahydropentalen-1-ol (7). A solution of 1 $(100 \mathrm{mg}, 0.833 \mathrm{mmol})$ in $\mathrm{Et}_{2} \mathrm{O}(6 \mathrm{~mL})$ was treated dropwise at $-78^{\circ} \mathrm{C}$ with $t-\mathrm{BuLi}(0.54$ $\mathrm{mL}, 0.92 \mathrm{mmol}, 1.7 \mathrm{M} /$ pentane, 1.1 equiv). The reaction mixture was stirred at $-78{ }^{\circ} \mathrm{C}$ for $10 \mathrm{~min}$, then at $\mathrm{rt}$ for $1 \mathrm{~h}$. The reaction mixture was recooled to $-78^{\circ} \mathrm{C}$ and treated rapidly with a solution of Davis' oxaziridine $(261 \mathrm{mg}, 1.00 \mathrm{mmol})$ in THF $(3 \mathrm{~mL})$. After 5 min at $-78{ }^{\circ} \mathrm{C}$ the reaction mixture was quenched with saturated aqueous $\mathrm{NaHCO}_{3}$ solution and extracted with $\mathrm{Et}_{2} \mathrm{O}$. The combined organic layers were dried $\left(\mathrm{MgSO}_{4}\right)$, filtered and concentrated. ${ }^{1} \mathrm{H}$ NMR of the crude product showed a dr $>20: 1$. Column chromatography on $\mathrm{SiO}_{2}$ (hexanes/EtOAc, 90:10) provided 7 (79 $\mathrm{mg}, 49 \%$ ) as a colorless oil. IR (neat) 3314, 2949, 2860, 1476, 1364, 1050, 1030, $1007 \mathrm{~cm}^{-1} ;{ }^{1} \mathrm{H}$ NMR $\delta 5.39$ (s, 1 H), 4.28 (s, $1 \mathrm{H}), 3.25$ (bt, $1 \mathrm{H}, J=7.2 \mathrm{~Hz}), 2.44$ (bt, $1 \mathrm{H}, J=7.2 \mathrm{~Hz}), 2.09$ (d, $1 \mathrm{H}, J=$ $13.6 \mathrm{~Hz}), 1.95(\mathrm{~d}, 1 \mathrm{H}, J=13.2 \mathrm{~Hz}), 1.76-1.67(\mathrm{~m}, 1 \mathrm{H}), 1.58-1.19(\mathrm{~m}, 6 \mathrm{H}), 0.91(\mathrm{~s}, 9$ $\mathrm{H}) ;{ }^{13} \mathrm{C}$ NMR $\delta 143.7,136.5,88.1,52.0,48.3,41.1,32.6,31.8,31.4,30.0,25.0$; MS (EI) $\mathrm{m} / z$ (rel. intensity) 177 ([M-OH] $\left.]^{+}, 100\right), 121$ (30); HRMS (EI) calcd for $\mathrm{C}_{13} \mathrm{H}_{21}(\mathrm{M}-\mathrm{OH})$ : 177.1647, found: 177.1643 .

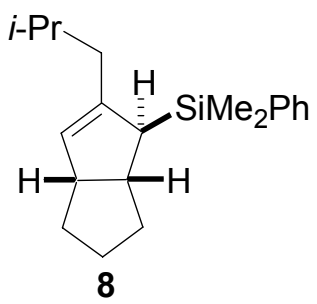

(2-Isobutyl-1,3a,4,5,6,6a-hexahydropentalen-1-yl)-dimethylphenylsilane (8). A solution of $1(250 \mathrm{mg}, 2.08 \mathrm{mmol})$ in $\mathrm{Et}_{2} \mathrm{O}(10 \mathrm{~mL})$ was treated dropwise at $-78{ }^{\circ} \mathrm{C}$ with $i$-PrLi ( $3.3 \mathrm{~mL}, 2.3 \mathrm{mmol}, 0.7 \mathrm{M} /$ pentane, 1.1 equiv). The reaction mixture was stirred at $-78{ }^{\circ} \mathrm{C}$ for $10 \mathrm{~min}$, then at $\mathrm{rt}$ for $2 \mathrm{~h}$. The reaction mixture was recooled to $-78^{\circ} \mathrm{C}$ and treated dropwise with $\mathrm{PhMe}_{2} \mathrm{SiCl}(0.350 \mathrm{~mL}, 2.08 \mathrm{mmol})$. After $45 \mathrm{~min}$ at $-78{ }^{\circ} \mathrm{C}$ the reaction mixture was quenched with saturated aqueous $\mathrm{NaHCO}_{3}$ solution and extracted with $\mathrm{Et}_{2} \mathrm{O}$. The combined organic layers were dried $\left(\mathrm{Na}_{2} \mathrm{SO}_{4}\right)$, filtered and concentrated. ${ }^{1} \mathrm{H}$ NMR of the crude product showed a dr $>20: 1$. Column chromatography on $\mathrm{SiO}_{2}$ (hexanes) provided $\mathbf{8}(431 \mathrm{mg}, 70 \%)$ as a colorless oil. IR (neat) 2945, 2860, 1427, 1243, 1110, 837, $697 \mathrm{~cm}^{-1} ;{ }^{1} \mathrm{H}$ NMR $\delta$ 7.54-7.52 (m, $\left.2 \mathrm{H}\right), 7.35-7.33(\mathrm{~m}, 3 \mathrm{H}), 4.99(\mathrm{~s}, 1 \mathrm{H})$, 2.93 (b, $1 \mathrm{H}), 2.55-2.49(\mathrm{~m}, 1 \mathrm{H}), 1.86(\mathrm{~d}, 1 \mathrm{H}, J=2.0 \mathrm{~Hz}), 1.76-1.67$ (m, $3 \mathrm{H}), 1.65-1.47$ (m, $3 \mathrm{H}), 1.41-1.33$ (m, $2 \mathrm{H}), 1.28-1.20(\mathrm{~m}, 1 \mathrm{H}), 0.74$ (d, $3 \mathrm{H}, J=6.4 \mathrm{~Hz}), 0.69$ (d, $3 \mathrm{H}, J$ $=6.8 \mathrm{~Hz}), 0.29(\mathrm{~s}, 3 \mathrm{H}), 0.27(\mathrm{~s}, 3 \mathrm{H}) ;{ }^{13} \mathrm{C}$ NMR $\delta 143.7,139.6,133.9,128.8,127.7$, $127.5,49.6,44.8,43.9,40.8,37.4,33.0,26.2,25.4$ 23.4, 21.5, -3.6, -3.9; MS (EI) $\mathrm{m} / \mathrm{z}$ (rel. intensity) $298\left(\mathrm{M}^{+}, 7\right), 162$ (25), 135 (100); HRMS (EI) calcd for $\mathrm{C}_{20} \mathrm{H}_{30} \mathrm{Si}$ : 298.2117, found: 298.2116 . 


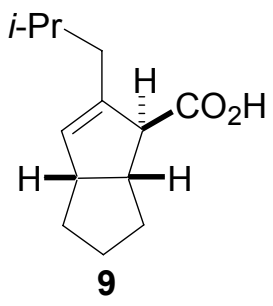

2-Isobutyl-1,3a,4,5,6,6a-hexahydropentalene-1-carboxylic acid (9). A solution of 1 $(20.0 \mathrm{mg}, 0.167 \mathrm{mmol})$ in $\mathrm{Et}_{2} \mathrm{O}(0.6 \mathrm{~mL})$ was treated dropwise at $-78{ }^{\circ} \mathrm{C}$ with $i$-PrLi $(0.260 \mathrm{~mL}, 0.182 \mathrm{mmol}, 0.7 \mathrm{M} /$ pentane, 1.1 equiv). The reaction mixture was stirred at $-78{ }^{\circ} \mathrm{C}$ for $10 \mathrm{~min}$, then at $\mathrm{rt}$ for $1 \mathrm{~h}$. The reaction mixture was cannulated into a slurry of $\mathrm{CO}_{2}$ in THF $(3 \mathrm{~mL})$ at $-78{ }^{\circ} \mathrm{C}$. The reaction mixture was allowed to warm to $\mathrm{rt}$ gradually over $3 \mathrm{~h}$, acidified with $10 \%$ aqueous $\mathrm{HCl}$ solution, and extracted with EtOAc. The combined organic layers were dried $\left(\mathrm{Na}_{2} \mathrm{SO}_{4}\right)$, filtered and concentrated. ${ }^{1} \mathrm{H}$ NMR of the crude product showed a $\mathrm{dr}=6.8: 1$. Column chromatography on $\mathrm{SiO}_{2}$ (hexanes/EtOAc, $85: 15)$ provided 9 (25 mg, 71\%) as a colorless oil. IR (neat) 2954, 2865, 1704, 1291, $1214 \mathrm{~cm}^{-1} ;{ }^{1} \mathrm{H}$ NMR $\delta 5.36(\mathrm{~s}, 1 \mathrm{H}), 3.28(\mathrm{~b}, 1 \mathrm{H}), 3.11(\mathrm{~b}, 1 \mathrm{H}), 2.91(\mathrm{bt}, 1 \mathrm{H}, J=7.6$ Hz), 2.01 (bd, $1 \mathrm{H}), 1.89$ (dd, $1 \mathrm{H}, J=8.8,13.6 \mathrm{~Hz}), 1.81-1.72(\mathrm{~m}, 2 \mathrm{H}), 1.65-1.58$ (m, 1 H), 1.55-1.38 (m, $4 \mathrm{H}), 0.91$ (d, $3 \mathrm{H}), 0.82$ (d, $3 \mathrm{H}) ;{ }^{13} \mathrm{C}$ NMR $\delta$ 182.0, 139.7, 133.9, 50.0, 46.3, 39.0, 34.9, 31.9, 26.3, 25.2, 23.4, 21.9; MS (EI) $m / z$ (rel. intensity) $208\left(\mathrm{M}^{+}, 65\right)$, 163 (100); HRMS (EI) calcd for $\mathrm{C}_{13} \mathrm{H}_{20} \mathrm{O}_{2}$ : 208.1463, found: 208.1463.

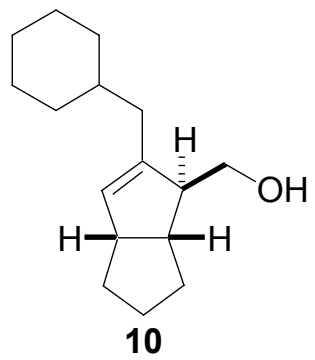

(2-Cyclohexylmethyl-1,3a,4,5,6,6a-hexahydropentalen-1-yl) methanol (10). A solution of cyclohexyl iodide $(0.260 \mathrm{~mL}, 2.01 \mathrm{mmol})$ in $\mathrm{Et}_{2} \mathrm{O}(2 \mathrm{~mL})$ was treated dropwise at $-78{ }^{\circ} \mathrm{C}$ with $t$-BuLi $(2.6 \mathrm{~mL}, 4.4 \mathrm{mmol}, 1.7 \mathrm{M} /$ pentane, 2.2 equiv). After 30 min at $-78{ }^{\circ} \mathrm{C}$ the reaction mixture was cannulated through a short filter of Celite into a new flask at $-78^{\circ} \mathrm{C}$. This clear solution of cyclohexyllithium was treated dropwise with a solution of $1(200 \mathrm{mg}, 1.67 \mathrm{mmol})$ in $\mathrm{Et}_{2} \mathrm{O}(5 \mathrm{~mL})$. The reaction mixture was stirred at $-78{ }^{\circ} \mathrm{C}$ for $10 \mathrm{~min}$, then at $\mathrm{rt}$ for $2 \mathrm{~h}$. The reaction mixture was recooled to $-78{ }^{\circ} \mathrm{C}$ and treated dropwise with a suspension of paraformaldehyde $(150 \mathrm{mg}, 5.01 \mathrm{mmol})$ in $\mathrm{Et}_{2} \mathrm{O}(5$ $\mathrm{mL}$ ). The reaction mixture was allowed to warm to $\mathrm{rt}$ gradually over $1 \mathrm{~h}$, quenched with saturated aqueous $\mathrm{NaHCO}_{3}$ solution and extracted with $\mathrm{Et}_{2} \mathrm{O}$. The combined organic layers were dried $\left(\mathrm{MgSO}_{4}\right)$, filtered and concentrated. ${ }^{1} \mathrm{H}$ NMR of the crude product showed a $\mathrm{dr}=11.4: 1$. Column chromatography on $\mathrm{SiO}_{2}$ (hexanes/EtOAc, 90:10) provided 10 (188 $\mathrm{mg}, 48 \%$ ) as a colorless oil. IR (neat) 3329, 2924, 2849, 1444, 1037 $\mathrm{cm}^{-1} ;{ }^{1} \mathrm{H}$ NMR $\delta 5.28(\mathrm{~s}, 1 \mathrm{H}), 3.65(\mathrm{dd}, 1 \mathrm{H}, J=2.8,10.4 \mathrm{~Hz}), 3.56(\mathrm{dd}, 1 \mathrm{H}, J=5.2$, $10.4 \mathrm{~Hz}$ ), 3.14-3.06 (m, $1 \mathrm{H}), 2.54-2.48$ (m, $1 \mathrm{H}), 2.38$ (b, $1 \mathrm{H}), 1.88$ (d, $2 \mathrm{H}, J=8.0 \mathrm{~Hz}$ ), 
1.79-1.56 (m, 7 H), 1.50-1.33 (m, 4 H), 1.28-1.08 (m, 5 H), 0.97-0.87 (m, 1 H), 0.83-0.73 (m, $1 \mathrm{H}) ;{ }^{13} \mathrm{C}$ NMR $\delta 140.9,132.7,64.9,58.0,49.1,45.4,37.0,36.0,35.3,34.3,32.9$, 32.4, 26.7, 26.5, 26.4, 25.3; MS (EI) $\mathrm{m} / z$ (rel. intensity) 234 (M+1 17), 203 (93), 121 (76), 107 (100); HRMS (EI) calcd for $\mathrm{C}_{16} \mathrm{H}_{26} \mathrm{O}: 234.1984$, found: 234.1981 .

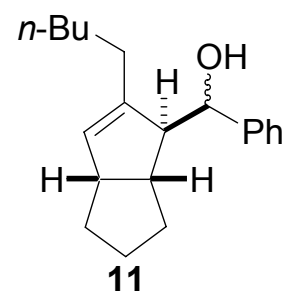

(2-Pentyl-1,3a,4,5,6,6a-hexahydropentalen-1-yl)-phenylmethanol (11). A solution of (-)-sparteine $(0.17 \mathrm{~mL}, 0.73 \mathrm{mmol})$ in hexanes $(4.5 \mathrm{~mL})$ was treated at $-78{ }^{\circ} \mathrm{C}$ with $n$ BuLi $(0.29 \mathrm{~mL}, 0.73 \mathrm{mmol}, 2.5 \mathrm{M} / \mathrm{h}$ exanes, 1.1 equiv). A solution of $1(80 \mathrm{mg}, 0.667$ mmol) in hexanes $(0.5 \mathrm{~mL})$ was added dropwise at $-78^{\circ} \mathrm{C}$. The reaction mixture was stirred at $-78{ }^{\circ} \mathrm{C}$ for $10 \mathrm{~min}$, then at $\mathrm{rt}$ for $2 \mathrm{~h}$. The reaction mixture was recooled to -78 ${ }^{\circ} \mathrm{C}$ and treated dropwise with $\mathrm{PhCHO}(0.08 \mathrm{~mL}, 0.80 \mathrm{mmol})$. After $30 \mathrm{~min}$ at $-78{ }^{\circ} \mathrm{C}$ the reaction mixture was warmed to $\mathrm{rt}$, quenched with saturated aqueous $\mathrm{NaHCO}_{3}$ solution and extracted with $\mathrm{Et}_{2} \mathrm{O}$. The combined organic layers were dried $\left(\mathrm{Na}_{2} \mathrm{SO}_{4}\right)$, filtered and concentrated. ${ }^{1} \mathrm{H}$ NMR of the crude product showed $\mathrm{a} d r=1: 1$. Column chromatography on $\mathrm{SiO}_{2}$ (hexanes/EtOAc, 95:5) provided two diastereomers of $\mathbf{1 1}$ [nonpolar diastereomer (11a): $67 \mathrm{mg}$, polar diastereomer (11b): $69 \mathrm{mg}$; 70\% combined yield] as colorless oils. Nonpolar diastereomer (11a): $[\alpha]_{\mathrm{D}}-0.31\left(c 0.23, \mathrm{CHCl}_{3}\right)$; IR (neat) 3422, 3026, 2933, 2859, 1450, $1062 \mathrm{~cm}^{-1}$; ${ }^{1} \mathrm{H}$ NMR $\delta$ 7.40-7.33 (m, $\left.4 \mathrm{H}\right), 7.26-7.22(\mathrm{~m}, 1 \mathrm{H}), 5.44(\mathrm{~s}, 1 \mathrm{H})$, $4.94(\mathrm{~s}, 1 \mathrm{H}), 3.06$ (b, $1 \mathrm{H}), 2.66(\mathrm{~s}, 1 \mathrm{H}), 2.54-2.48(\mathrm{~m}, 1 \mathrm{H}), 2.17-2.09(\mathrm{~m}, 1 \mathrm{H}), 2.05-$ $1.98(\mathrm{~m}, 1 \mathrm{H}), 1.82(\mathrm{~d}, 1 \mathrm{H}, J=1.6 \mathrm{~Hz}), 1.60-1.45(\mathrm{~m}, 4 \mathrm{H}), 1.40-1.28(\mathrm{~m}, 8 \mathrm{H}), 0.92(\mathrm{t}, 3$ $\mathrm{H}, J=6.8 \mathrm{~Hz}) ;{ }^{13} \mathrm{C}$ NMR $\delta 143.2,142.9,132.6,128.2,126.8,125.8,72.2,63.2,49.1$, 41.0, 35.2, 32.4, 31.8, 28.9, 27.6, 25.2, 22.7, 14.2; MS (CI) m/z (rel. intensity) 307 ([M+Na $\left.]^{+}, 63\right), 267$ (100); HRMS (CI) calcd for $\mathrm{C}_{20} \mathrm{H}_{28} \mathrm{ONa}(\mathrm{M}+\mathrm{Na})$ : 307.2038, found: 307.2037. Polar diastereomer (11b): $[\alpha]_{\mathrm{D}}+12.6\left(c 0.140, \mathrm{CHCl}_{3}\right)$; IR (neat) 3397, 3023, 2929, 2862, 1452, $1034 \mathrm{~cm}^{-1} ;{ }^{1} \mathrm{H}$ NMR $\delta$ 7.35-7.29 (m, $\left.4 \mathrm{H}\right), 7.26-7.21(\mathrm{~m}, 1 \mathrm{H}), 5.23(\mathrm{~s}$, $1 \mathrm{H}), 4.80(\mathrm{t}, 1 \mathrm{H}, J=4.8 \mathrm{~Hz}), 2.81(\mathrm{~s}, 1 \mathrm{H}), 2.75(\mathrm{~b}, 1 \mathrm{H}), 2.47-2.41(\mathrm{~m}, 1 \mathrm{H}), 2.03-1.95$ $(\mathrm{m}, 1 \mathrm{H}), 1.90(\mathrm{~d}, 1 \mathrm{H}, J=5.2 \mathrm{~Hz}), 1.85-1.75(\mathrm{~m}, 1 \mathrm{H}), 1.74-1.65(\mathrm{~m}, 1 \mathrm{H}), 1.59-1.51(\mathrm{~m}$, $1 \mathrm{H}), 1.47-1.09(\mathrm{~m}, 10 \mathrm{H}), 0.85(\mathrm{t}, 3 \mathrm{H}, J=7.2 \mathrm{~Hz}) ;{ }^{13} \mathrm{C}$ NMR $\delta 143.6,143.2,132.6$, 128.0, 127.0, 126.2, 76.7, 62.2, 48.7, 45.4, 35.1, 32.0, 31.7, 30.6, 27.6, 25.3, 22.6, 14.2; MS (CI) $\mathrm{m} / z$ (rel. intensity) $307\left([\mathrm{M}+\mathrm{Na}]^{+}, 60\right), 267$ (100); HRMS (CI) calcd for $\mathrm{C}_{20} \mathrm{H}_{28} \mathrm{ONa}(\mathrm{M}+\mathrm{Na}): 307.2038$, found: 307.2034 . Formation of the (S)-MTPA ester of each diastereomer [(S)-MTPA chloride, $\left.\mathrm{Et}_{3} \mathrm{~N}, \mathrm{CH}_{2} \mathrm{Cl}_{2}\right]$ and analysis of the crude ${ }^{1} \mathrm{H} N \mathrm{NR}$ spectra showed enantiomeric excesses of $9 \%$ and $16 \%$ for the $11 \mathbf{a}$ and $11 \mathbf{b}$, respectively. 


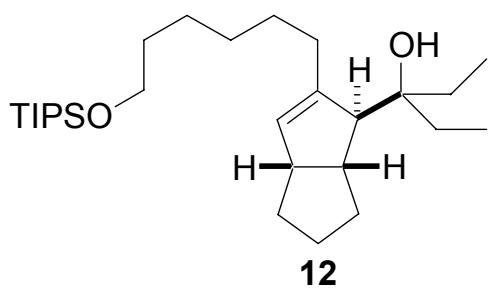

3-[2-(6-Triisopropylsilanyloxyhexyl)-1,3a,4,5,6,6a-hexahydropentalen-1-yl] pentan3-ol (12). A solution of 5-(triisopropylsilanyloxy)pentyl iodide (400 mg, $1.08 \mathrm{mmol})$ in hexanes $(2 \mathrm{~mL})$ and $\mathrm{Et}_{2} \mathrm{O}(0.05 \mathrm{~mL})$ was treated dropwise at $-78{ }^{\circ} \mathrm{C}$ with $t$-BuLi $(1.40$ $\mathrm{mL}, 2.38 \mathrm{mmol}, 1.7 \mathrm{M} /$ pentane, 2.2 equiv). After $25 \mathrm{~min}$ at $-78^{\circ} \mathrm{C}$ the reaction mixture was cannulated through a short filter of Celite into a new flask with hexanes $(1 \mathrm{~mL})$ at $78^{\circ} \mathrm{C}$. (-)-Sparteine $(0.23 \mathrm{~mL}, 0.98 \mathrm{mmol})$ was added, followed by dropwise addition of a solution of $1(118 \mathrm{mg}, 0.983 \mathrm{mmol})$ in hexanes $(0.5 \mathrm{~mL})$. The reaction mixture was stirred at $-78{ }^{\circ} \mathrm{C}$ for $10 \mathrm{~min}$, then at $0{ }^{\circ} \mathrm{C}$ for $2 \mathrm{~h}$. The reaction mixture was recooled to $78{ }^{\circ} \mathrm{C}$ and treated dropwise with 3-pentanone $(0.2 \mathrm{~mL})$. The reaction mixture was allowed to warm to rt gradually over 30 min, quenched with saturated aqueous $\mathrm{NaHCO}_{3}$ solution and extracted with $\mathrm{Et}_{2} \mathrm{O}$. The combined organic layers were dried $\left(\mathrm{MgSO}_{4}\right)$, filtered and concentrated. ${ }^{1} \mathrm{H}$ NMR of the crude product showed a $\mathrm{dr}=9.3: 1$. Column chromatography on $\mathrm{SiO}_{2}$ (hexanes/Et $2 \mathrm{O}, 98: 2$ ) provided $\mathbf{1 2}(257 \mathrm{mg}, 58 \%)$ as a colorless oil. IR (neat) 3489, 2936, 2862, 1463, $1104 \mathrm{~cm}^{-1} ;{ }^{1} \mathrm{H}$ NMR $\delta 5.27$ (s, $\left.1 \mathrm{H}\right), 3.66$ (t, $2 \mathrm{H}, J$ $=6.4 \mathrm{~Hz}), 3.00(\mathrm{~b}, 1 \mathrm{H}), 2.48(\mathrm{~s}, 1 \mathrm{H}), 2.36-2.30(\mathrm{~m}, 1 \mathrm{H}), 2.22-2.14(\mathrm{~m}, 1 \mathrm{H}), 2.08-2.01$ $(\mathrm{m}, 1 \mathrm{H}), 1.83-1.74(\mathrm{~m}, 1 \mathrm{H}), 1.68-1.59(\mathrm{~m}, 1 \mathrm{H}), 1.58-1.20(\mathrm{~m}, 16 \mathrm{H}), 1.16(\mathrm{~s}, 1 \mathrm{H})$, 1.11-1.01 (m, $21 \mathrm{H}), 0.92(\mathrm{t}, 3 \mathrm{H}, J=7.2 \mathrm{~Hz}), 0.87(\mathrm{t}, 3 \mathrm{H}, J=7.2 \mathrm{~Hz}) ;{ }^{13} \mathrm{C}$ NMR $\delta$ 144.1, 132.2, 77.4, 63.7, 62.5, 48.8, 44.9, 35.8, 33.2, 32.2, 32.0, 30.2, 29.5, 28.54, 28.48, 25.9, 25.6, 18.2, 12.1, 7.84, 7.78; MS (EI) $\mathrm{m} / z$ (rel. intensity) 407 ([M- $\left.\left.\mathrm{C}_{3} \mathrm{H}_{7}\right]^{+}, 10\right), 321$ (70); HRMS (EI) calcd for $\mathrm{C}_{26} \mathrm{H}_{49} \mathrm{O}_{2} \mathrm{Si}\left(\mathrm{M}-\mathrm{C}_{2} \mathrm{H}_{5}\right)$ : 421.3502, found: 421.3497 .

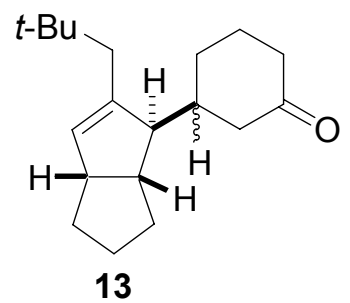

3-[2-(2,2-Dimethylpropyl)-1,3a,4,5,6,6a-hexahydropentalen-1-yl]cyclohexanone (13). A solution of $1(40 \mathrm{mg}, 0.33 \mathrm{mmol})$ in $\mathrm{Et}_{2} \mathrm{O}(1.8 \mathrm{~mL})$ was treated dropwise at $-78{ }^{\circ} \mathrm{C}$ with $t$-BuLi $(0.22 \mathrm{~mL}, 0.37 \mathrm{mmol}, 1.7 \mathrm{M} /$ pentane, 1.1 equiv). The reaction mixture was stirred at $-78{ }^{\circ} \mathrm{C}$ for $10 \mathrm{~min}$, then at $\mathrm{rt}$ for $1 \mathrm{~h}$. The reaction mixture was recooled to -78 ${ }^{\circ} \mathrm{C}$ and treated with a solution of $\mathrm{CuCN}$ (30 mg, 1 equiv) and $\mathrm{LiCl}(28 \mathrm{mg}, 2$ equiv) in THF $(1.5 \mathrm{~mL})$. After $10 \mathrm{~min}$ a solution of cyclohexanone $(0.035 \mathrm{~mL}, 0.37 \mathrm{mmol})$ and TMSCl $(0.10 \mathrm{~mL})$ in $\mathrm{Et}_{2} \mathrm{O}(1.5 \mathrm{~mL})$ was added dropwise. After $1.5 \mathrm{~h}$ at $-78{ }^{\circ} \mathrm{C}$ the reaction mixture was warmed to $\mathrm{rt}$, quenched with saturated aqueous $\mathrm{NaHCO}_{3}$ solution and extracted with $\mathrm{Et}_{2} \mathrm{O}$. The combined organic layers were dried $\left(\mathrm{MgSO}_{4}\right)$, filtered and concentrated. ${ }^{1} \mathrm{H}$ NMR of the crude product showed a $\mathrm{dr}=2.5: 1$. Column 
chromatography on $\mathrm{SiO}_{2}$ (hexanes/EtOAc, 90:10) provided $13(57 \mathrm{mg}, 63 \%)$ as an inseparable 2.5:1 mixture of diastereomers and a colorless oil. IR (neat) 2949, 2861, $1715,1443,1359,1223 \mathrm{~cm}^{-1}$; ${ }^{1} \mathrm{H}$ NMR $\delta 5.19(\mathrm{~s}, 1 \mathrm{H}), 2.96(\mathrm{~b}, 1 \mathrm{H}), 2.44-2.20(\mathrm{~m}, 4 \mathrm{H})$, 2.12-2.03 (m, 2 H), 1.97-1.87 (m, 2 H), 1.78-1.54 (m, 6 H), 1.52-1.45 (m, 2 H), 1.43-1.23 $(\mathrm{m}, 3 \mathrm{H}), 0.88(\mathrm{~s}, 9 \mathrm{H}) ;{ }^{13} \mathrm{C}$ NMR $\delta 213.2,141.3,133.5,61.7,49.5,42.53,42.45,41.9$, 41.7, 40.9, 40.2, 36.1, 32.6, 31.9, 30.2, 25.9, 25.5; MS (CI) m/z (rel. intensity) 297 $\left([\mathrm{M}+\mathrm{Na}]^{+}, 100\right)$; HRMS (CI) calcd for $\mathrm{C}_{19} \mathrm{H}_{30} \mathrm{ONa}(\mathrm{M}+\mathrm{Na})$ : 297.2194, found: 297.2199.

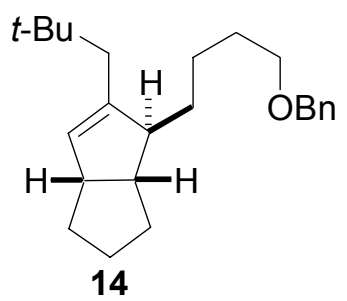

4-(4-Benzyloxybutyl)-5-(2,2-dimethylpropyl)-1,2,3,3a,4,6a-hexahydropentalene (14). A solution of $1(150 \mathrm{mg}, 1.25 \mathrm{mmol})$ in $\mathrm{Et}_{2} \mathrm{O}(7.5 \mathrm{~mL})$ was treated dropwise at $-78{ }^{\circ} \mathrm{C}$ with $t$-BuLi $(0.810 \mathrm{~mL}, 1.38 \mathrm{mmol}, 1.7 \mathrm{M} /$ pentane, 1.1 equiv $)$. The reaction mixture was stirred at $-78{ }^{\circ} \mathrm{C}$ for $10 \mathrm{~min}$, then at $\mathrm{rt}$ for $2 \mathrm{~h}$. The reaction mixture was recooled to -78 ${ }^{\circ} \mathrm{C}$ and treated with a solution of $\mathrm{CuCN}$ (112 mg, 1 equiv) and $\mathrm{LiCl}(106 \mathrm{mg}, 2$ equiv) in THF (5 mL). After 20 min a solution of 4-(benzyloxy)butyl iodide (435 mg, $1.50 \mathrm{mmol})$ in THF $(2 \mathrm{~mL})$ was added dropwise. After $20 \mathrm{~min}$ at $-78{ }^{\circ} \mathrm{C}$ the reaction mixture was warmed to rt overnight, quenched with saturated aqueous $\mathrm{NaHCO}_{3}$ solution and extracted with $\mathrm{Et}_{2} \mathrm{O}$. The combined organic layers were dried $\left(\mathrm{MgSO}_{4}\right)$, filtered and concentrated. ${ }^{1} \mathrm{H}$ NMR of the crude product showed a $\mathrm{dr}=4.7: 1$. Column chromatography on $\mathrm{SiO}_{2}$ (hexanes/ $\left.\mathrm{Et}_{2} \mathrm{O}, 85: 15\right)$ provided $\mathbf{1 4}(194 \mathrm{mg}, 46 \%)$ as an inseparable 4.7:1 mixture of diastereomers and a colorless oil. IR (neat) 2937, 2858, 1453, 1362, $1098 \mathrm{~cm}^{-1} ;{ }^{1} \mathrm{H}$ NMR $\delta$ 7.35-7.26 (m, $5 \mathrm{H}), 5.11(\mathrm{~s}, 1 \mathrm{H}), 4.51(\mathrm{~s}, 2 \mathrm{H}), 3.47(\mathrm{t}, 2 \mathrm{H}, J=6.4 \mathrm{~Hz}), 3.08-3.03(\mathrm{~m}$, $1 \mathrm{H}), 2.31-2.25(\mathrm{~m}, 1 \mathrm{H}), 2.22$ (bd, $1 \mathrm{H}, J=9.2 \mathrm{~Hz}), 1.91-1.82(\mathrm{~m}, 2 \mathrm{H}), 1.73-1.50$ (m, 4 H), 1.49-1.20 (m, $6 \mathrm{H}), 1.15-1.04$ (m, $2 \mathrm{H}), 0.89$ (s, $9 \mathrm{H}) ;{ }^{13} \mathrm{C}$ NMR $\delta 144.5,138.8,131.8$, $128.5,128.0,127.9,127.8,127.6,85.3,73.0,70.6,69.0,56.9,48.8,47.5,42.2,35.6,34.2$, 32.6, 30.3, 29.8, 25.5, 23.8, 19.2, 13.9; MS (EI) $\mathrm{m} / z$ (rel. intensity) $340\left(\mathrm{M}^{+}, 5\right), 249$ (15), 163 (83), 107 (100); HRMS (EI) calcd for $\mathrm{C}_{24} \mathrm{H}_{36} \mathrm{O}: 340.2766$, found: 340.2756 .

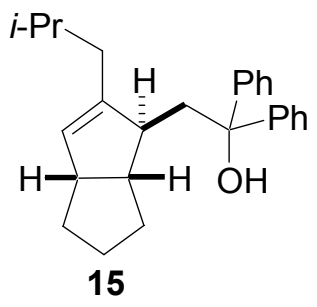

2-(2-Isobutyl-1,3a,4,5,6,6a-hexahydropentalen-1-yl)-1,1-diphenylethanol (15). A solution of $1(400 \mathrm{mg}, 3.33 \mathrm{mmol})$ in $\mathrm{Et}_{2} \mathrm{O}(17 \mathrm{~mL})$ was treated dropwise at $-78{ }^{\circ} \mathrm{C}$ with $i$-PrLi $(5.20 \mathrm{~mL}, 3.64 \mathrm{mmol}, 0.7 \mathrm{M} /$ pentane, 1.1 equiv). The reaction mixture was stirred at $-78{ }^{\circ} \mathrm{C}$ for $10 \mathrm{~min}$, then at $\mathrm{rt}$ for $1 \mathrm{~h}$. The reaction mixture was recooled to $-78{ }^{\circ} \mathrm{C}$ and 
treated with a solution of $\mathrm{CuCN}$ (298 $\mathrm{mg}, 1$ equiv) and $\mathrm{LiCl}(282 \mathrm{mg}, 2$ equiv) in THF $(10 \mathrm{~mL})$. After $10 \mathrm{~min}$ a solution of 1,1-diphenyloxirane $(653 \mathrm{mg}, 3.33 \mathrm{mmol})$ in THF (4 $\mathrm{mL}$ ) was added dropwise. The reaction mixture was warmed to $\mathrm{rt}$ overnight, quenched with saturated aqueous $\mathrm{NaHCO}_{3}$ solution and extracted with $\mathrm{Et}_{2} \mathrm{O}$. The combined organic layers were dried $\left(\mathrm{MgSO}_{4}\right)$, filtered and concentrated. ${ }^{1} \mathrm{H}$ NMR of the crude product showed a dr = 9.7:1. Column chromatography on $\mathrm{SiO}_{2}$ (hexanes/EtOAc, 95:5) provided 15 (731 mg, 61\%) as a colorless oil. IR (neat) 3560, 3022, 2948, 2866, 1493, 1444, 1168, 1053, $700 \mathrm{~cm}^{-1} ;{ }^{1} \mathrm{H}$ NMR $\delta$ 7.49-7.45 (m, $4 \mathrm{H}$ ), 7.36-7.29 (m, $\left.4 \mathrm{H}\right), 7.26-7.21$ (m, $2 \mathrm{H}), 5.11(\mathrm{~s}, 1 \mathrm{H}), 3.12$ (bt, $1 \mathrm{H}, J=7.6 \mathrm{~Hz}), 2.55$ (d, $1 \mathrm{H}, J=12.8 \mathrm{~Hz}), 2.30-2.17$ (m, $3 \mathrm{H}), 2.10(\mathrm{~s}, 1 \mathrm{H}), 1.95-1.87(\mathrm{~m}, 1 \mathrm{H}), 1.78(\mathrm{dd}, 1 \mathrm{H}, J=9.6,14.0 \mathrm{~Hz}), 1.60-1.45$ (m, $2 \mathrm{H}), 1.42-1.30$ (m, $3 \mathrm{H}), 1.20-1.15(\mathrm{~m}, 1 \mathrm{H}), 0.83(\mathrm{~d}, 3 \mathrm{H}, J=6.4 \mathrm{~Hz}), 0.73(\mathrm{~d}, 3 \mathrm{H}, J=$ $6.4 \mathrm{~Hz}) ;{ }^{13} \mathrm{C}$ NMR $\delta 148.0,147.3,145.5,129.9,128.2,126.9,126.8,126.32,126.29$, 78.9, 50.1, 49.0, 46.1, 38.3, 34.9, 32.4, 26.1, 25.4, 23.6, 21.8; MS (EI) $\mathrm{m} / \mathrm{z}$ (rel. intensity) 359 ([M-H] $\left.]^{+}, 13\right), 342$ (57), 163 (100); HRMS (EI) calcd for $\mathrm{C}_{26} \mathrm{H}_{31} \mathrm{O}(\mathrm{M}-\mathrm{H})$ : 359.2375 , found: 359.2379 .

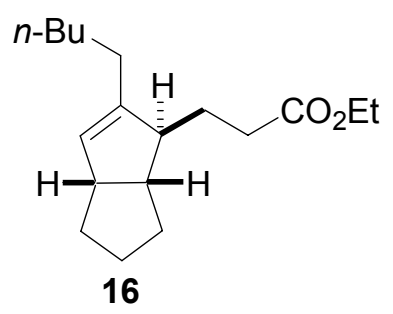

3-(2-Pentyl-1,3a,4,5,6,6a-hexahydropentalen-1-yl)propionic acid ethyl ester (16). A solution of (-)-sparteine $(0.380 \mathrm{~mL}, 1.67 \mathrm{mmol})$ in hexanes $(4 \mathrm{~mL})$ was treated at $-78{ }^{\circ} \mathrm{C}$ with $n$-BuLi ( $0.740 \mathrm{~mL}, 1.85 \mathrm{mmol}, 2.5 \mathrm{M} /$ hexanes, 1.1 equiv). A solution of $1(200 \mathrm{mg}$, $1.67 \mathrm{mmol})$ in hexanes $(0.5 \mathrm{~mL})$ was added dropwise at $-78^{\circ} \mathrm{C}$. The reaction mixture was stirred at $-78^{\circ} \mathrm{C}$ for $10 \mathrm{~min}$, then at $\mathrm{rt}$ for $1 \mathrm{~h}$ and $40 \mathrm{~min}$. The reaction mixture was recooled to $-78^{\circ} \mathrm{C}$ and treated with a solution of $\mathrm{CuCN}$ (149 $\mathrm{mg}, 1$ equiv) and $\mathrm{LiCl}(140$ $\mathrm{mg}, 2$ equiv) in THF $(5 \mathrm{~mL})$. After $10 \mathrm{~min}$ a solution of ethyl acrylate $(0.50 \mathrm{~mL}, 0.30$ $\mathrm{mmol})$ and TMSCl $(0.26 \mathrm{~mL}, 2.05 \mathrm{mmol})$ in THF $(3 \mathrm{~mL})$ was added dropwise. After warming gradually to rt over $2 \mathrm{~h}$, the reaction mixture was quenched with saturated aqueous $\mathrm{NaHCO}_{3}$ solution and extracted with $\mathrm{Et}_{2} \mathrm{O}$. The combined organic layers were dried $\left(\mathrm{MgSO}_{4}\right)$, filtered and concentrated. ${ }^{1} \mathrm{H}$ NMR of the crude product showed a dr $=$ 8.4:1. Column chromatography on $\mathrm{SiO}_{2}$ (hexanes/Et $/ \mathrm{E}_{2} \mathrm{O}, 98: 2$ ) provided 16 (325 mg, $70 \%$ ) as a colorless oil. IR (neat) $2933,2856,1736,1442,1368,1160,1037 \mathrm{~cm}^{-1} ;{ }^{1} \mathrm{H}$ NMR $\delta 5.11(\mathrm{~s}, 1 \mathrm{H}), 4.10(\mathrm{q}, 2 \mathrm{H}, J=7.2 \mathrm{~Hz}), 3.05-3.01(\mathrm{~m}, 1 \mathrm{H}), 2.35-2.15(\mathrm{~m}, 4 \mathrm{H})$, 2.02-1.82 (m, $3 \mathrm{H}), 1.71-1.62(\mathrm{~m}, 1 \mathrm{H}), 1.59-1.49(\mathrm{~m}, 1 \mathrm{H}), 1.48-1.19(\mathrm{~m}, 11 \mathrm{H}), 1.23(\mathrm{t}$, $3 \mathrm{H}, J=7.2 \mathrm{~Hz}$ ), 0.86 (t, $3 \mathrm{H}, J=7.2 \mathrm{~Hz}) ;{ }^{13} \mathrm{C}$ NMR $\delta 174.2,145.7,128.3,60.3,54.6$, 48.9, 47.1, 35.4, 32.4, 31.84, 31.77, 29.0, 28.9, 27.3, 25.3, 22.6, 14.3, 14.2; MS (EI) $\mathrm{m} / \mathrm{z}$ (rel. intensity) $278\left(\mathrm{M}^{+}, 100\right), 159$ (55), 113 (83); HRMS (EI) calcd for $\mathrm{C}_{18} \mathrm{H}_{30} \mathrm{O}_{2}$ : 278.2246, found: 278.2239 . 


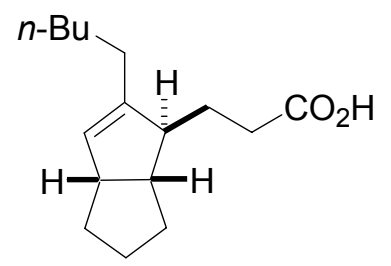

3-(2-Pentyl-1,3a,4,5,6,6a-hexahydropentalen-1-yl)propionic acid. A solution of 16 $(320 \mathrm{mg}, 1.15 \mathrm{mmol})$ in THF $(10 \mathrm{~mL}), \mathrm{MeOH}(2 \mathrm{~mL})$, and $\mathrm{H}_{2} \mathrm{O}(10 \mathrm{~mL})$ was treated with $\mathrm{LiOH} \cdot \mathrm{H}_{2} \mathrm{O}(240 \mathrm{mg})$. The reaction mixture was stirred at $\mathrm{rt}$ for $1 \mathrm{~h}$, acidified to $\mathrm{pH}=2$ with conc. $\mathrm{HCl}$, and extracted with $\mathrm{Et}_{2} \mathrm{O}$. The combined $\mathrm{Et}_{2} \mathrm{O}$ extracts were dried $\left(\mathrm{MgSO}_{4}\right)$, filtered and concentrated. Column chromatography on $\mathrm{SiO}_{2}$ (hexanes/EtOAc, 80:20) provided 3-(2-pentyl-1,3a,4,5,6,6a-hexahydropentalen-1-yl)propionic acid (270 $\mathrm{mg}, 94 \%$ ) as a colorless oil. IR (neat) $3035,2933,2859,2664,1708,1446,1413,1283$, 1242, $923 \mathrm{~cm}^{-1}$; ${ }^{1} \mathrm{H}$ NMR $\delta 5.14(\mathrm{~s}, 1 \mathrm{H}), 3.10-3.02(\mathrm{~m}, 1 \mathrm{H}), 2.42-2.18(\mathrm{~m}, 4 \mathrm{H}), 2.03-$ $1.85(\mathrm{~m}, 3 \mathrm{H}), 1.74-1.65(\mathrm{~m}, 1 \mathrm{H}), 1.62-1.19(\mathrm{~m}, 12 \mathrm{H}), 0.88(\mathrm{t}, 3 \mathrm{H}, J=6.8 \mathrm{~Hz}) ;{ }^{13} \mathrm{C}$ NMR $\delta 181.2,145.5,128.6,54.6,49.0,47.1,35.5,32.4,31.8,31.6,28.9,28.8,27.4$, 25.3, 22.7, 14.2; MS (EI) $\mathrm{m} / \mathrm{z}$ (rel. intensity) $250\left(\mathrm{M}^{+}, 100\right)$; HRMS (EI) calcd for $\mathrm{C}_{16} \mathrm{H}_{26} \mathrm{O}_{2}$ : 250.1933 , found: 250.1935 .

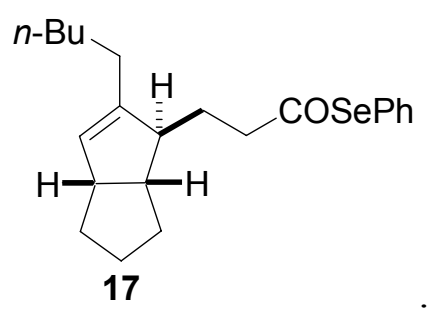

3-(2-Pentyl-1,3a,4,5,6,6a-hexahydropentalen-1-yl)propionic acid phenylselenyl ester (17). A solution of 3-(2-pentyl-1,3a,4,5,6,6a-hexahydropentalen-1-yl)propionic acid (267 $\mathrm{mg}, 1.07 \mathrm{mmol})$ and tri- $n$-butyl phosphine $(0.53 \mathrm{~mL}, 2.14 \mathrm{mmol})$ in THF $(10 \mathrm{~mL})$ was treated in one portion with $N$-(phenylselenyl)phthalimide $(647 \mathrm{mg}, 2.14 \mathrm{mmol})$. The reaction mixture was stirred at $\mathrm{rt}$ for $16 \mathrm{~h}$ and concentrated. Column chromatography on $\mathrm{SiO}_{2}$ (hexanes/ $\left.\mathrm{Et}_{2} \mathrm{O}, 99: 1\right)$ provided $17(362 \mathrm{mg}, 87 \%)$ as a light yellow oil. IR (neat) $3059,2930,2855,1723,1578,1478,1436,1020,733,687 \mathrm{~cm}^{-1} ;{ }^{1} \mathrm{H}$ NMR $\delta 7.53-7.51$ (m, $2 \mathrm{H}), 7.39-7.36(\mathrm{~m}, 3 \mathrm{H}), 5.16(\mathrm{~s}, 1 \mathrm{H}), 3.10-3.03(\mathrm{~m}, 1 \mathrm{H}), 2.76-2.62(\mathrm{~m}, 2 \mathrm{H}), 2.28-$ $2.20(\mathrm{~m}, 2 \mathrm{H}), 2.03-1.92(\mathrm{~m}, 2 \mathrm{H}), 1.93-1.84(\mathrm{~m}, 1 \mathrm{H}), 1.75-1.66(\mathrm{~m}, 1 \mathrm{H}), 1.63-1.52(\mathrm{~m}$, $2 \mathrm{H}), 1.50-1.20(\mathrm{~m}, 10 \mathrm{H}), 0.90(\mathrm{t}, 3 \mathrm{H}, J=6.8 \mathrm{~Hz}) ;{ }^{13} \mathrm{C}$ NMR $\delta 200.8,145.3,135.9$, 129.4, 128.9, 128.8, 126.7, 54.3, 49.0, 47.1, 45.1, 35.4, 32.4, 31.8, 29.3, 28.9, 27.4, 25.3, 22.7, 14.2; MS (EI) $\mathrm{m} / \mathrm{z}$ (rel. intensity) $391\left([\mathrm{M}+\mathrm{H}]^{+}, 25\right), 233$ (100); HRMS (EI) calcd for $\mathrm{C}_{22} \mathrm{H}_{31} \mathrm{OSe}(\mathrm{M}+\mathrm{H})$ : 391.1540 , found: 391.1547 . 


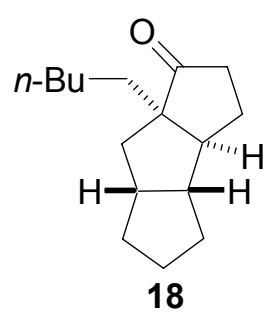

7a-Pentyl-decahydrocyclopenta[a]pentalen-1-one (18). A solution of 17 (145 $\mathrm{mg}$, $0.373 \mathrm{mmol})$ and $\operatorname{AIBN}(6.0 \mathrm{mg}, 0.037 \mathrm{mmol})$ in benzene $(35 \mathrm{~mL})$ was placed in a preheated oil bath $\left(85^{\circ} \mathrm{C}\right)$ and treated dropwise via syringe pump with a solution of $\mathrm{Bu}_{3} \mathrm{SnH}(0.120 \mathrm{~mL}, 0.448 \mathrm{mmol})$ in benzene $(3.8 \mathrm{~mL})$ over $1 \mathrm{~h}$. The reaction mixture was stirred an additional $30 \mathrm{~min}$ at $85{ }^{\circ} \mathrm{C}$, cooled to rt, and concentrated. ${ }^{13} \mathrm{C}$ NMR of the crude product mixture showed a single diastereomer. Column chromatography on $\mathrm{SiO}_{2}$ (hexanes/ $\left.\mathrm{Et}_{2} \mathrm{O}, 95: 5 \rightarrow 90: 10\right)$ provided 18 (71 mg, 81\%) as a light yellow oil. IR (neat) 2934, 2856, 1735, 1459, 1408, $1101 \mathrm{~cm}^{-1}$; ${ }^{1} \mathrm{H}$ NMR $\delta$ 2.34-2.24 (m, $\left.3 \mathrm{H}\right), 2.23-2.17$ (m, 1 H), 2.12-2.05 (m, 2 H), 2.02-1.95 (m, $1 \mathrm{H}), 1.80-1.67$ (m, $2 \mathrm{H}), 1.63-1.55$ (m, $2 \mathrm{H}), 1.52-$ $1.48(\mathrm{~m}, 1 \mathrm{H}), 1.46-1.37(\mathrm{~m}, 3 \mathrm{H}), 1.35-1.10(\mathrm{~m}, 8 \mathrm{H}), 0.85(\mathrm{t}, 3 \mathrm{H}, J=7.2 \mathrm{~Hz}) ;{ }^{13} \mathrm{C} \mathrm{NMR}$ $\delta 225.3,63.0,53.6,51.9,43.0,42.2,36.4,35.7,33.6,33.4,32.5,25.6,25.0,24.8,22.6$, 14.2; MS (EI) $m / z$ (rel. intensity) $235\left([\mathrm{M}+\mathrm{H}]^{+}, 100\right), 217$ (80), 164 (98); HRMS (EI) calcd for $\mathrm{C}_{16} \mathrm{H}_{27} \mathrm{O}(\mathrm{M}+\mathrm{H})$ : 235.2062, found: 235.2066 .

Proof of stereochemistry of 18: Ester 16 was reduced to 3-(2-pentyl-1,3a,4,5,6,6ahexahydropentalen-1-yl)propan-1-ol as outlined below. ${ }^{1} \mathrm{H}$ NMR, COSY and NOESY data established the relative stereochemistry of this alcohol. Given the precedent for 5exo acyl- and vinyl-radical cyclizations of cyclopentenyl and cyclohexenyl systems to form exclusively cis-annulation products, ${ }^{1-3}$ it can be concluded that triquinane 18 possesses cis-transoid-cis stereochemistry.

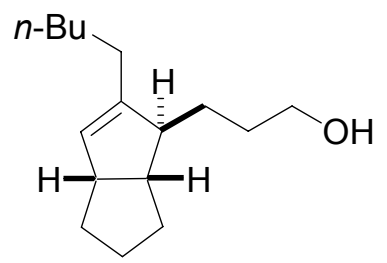

3-(2-Pentyl-1,3a,4,5,6,6a-hexahydropentalen-1-yl)propan-1-ol. A solution of 16 (200 $\mathrm{mg}, 0.719 \mathrm{mmol})$ in THF $(25 \mathrm{~mL})$ was treated with $\mathrm{LiBH}_{4}(220 \mathrm{mg})$. The reaction mixture was stirred at $\mathrm{rt}$ for $2 \mathrm{~h}$, quenched with brine followed by $10 \%$ aqueous $\mathrm{HCl}$, and extracted with $\mathrm{Et}_{2} \mathrm{O}$. The combined $\mathrm{Et}_{2} \mathrm{O}$ extracts were dried $\left(\mathrm{MgSO}_{4}\right)$, filtered and concentrated. Column chromatography on $\mathrm{SiO}_{2}$ (hexanes/EtOAc, 80:20) provided 3-(2pentyl-1,3a,4,5,6,6a-hexahydropentalen-1-yl)propan-1-ol (124 mg, 73\%) as a colorless oil. IR (neat) 3325, 2928, 2857, 1448, 1380, $1059 \mathrm{~cm}^{-1}$; ${ }^{1} \mathrm{H}$ NMR $\delta 5.11(\mathrm{~s}, 1 \mathrm{H}), 3.65$ (t, $2 \mathrm{H}, J=6.0 \mathrm{~Hz}$ ), 3.07-3.03 (m, $1 \mathrm{H}), 2.31-2.26$ (m, $1 \mathrm{H}), 2.17$ (bd, $1 \mathrm{H}, J=8.8 \mathrm{~Hz}), 2.02-$ $1.94(\mathrm{~m}, 1 \mathrm{H}), 1.91-1.84(\mathrm{~m}, 1 \mathrm{H}), 1.73-1.13(17 \mathrm{H}), 0.88(\mathrm{t}, 3 \mathrm{H}, J=7.2 \mathrm{~Hz}) ;{ }^{13} \mathrm{C} \mathrm{NMR} \delta$ $146.4,127.8,63.6,55.2$, 49.0, 47.6, 35.6, 32.6, 31.9, 30.4, 30.1, 29.0, 27.5, 25.4, 22.7, 14.2; MS (EI) $m / z$ (rel. intensity) $236\left(\mathrm{M}^{+}, 65\right), 219$ (100); HRMS (EI) calcd for $\mathrm{C}_{16} \mathrm{H}_{28} \mathrm{O}$ : 236.2140, found: 236.2146 . 


\section{References}

(1) Stork, G.; Baine, N. H. J. Am. Chem. Soc. 1982, 104, 2321.

(2) Fernandez-Mateos, A.; Coca, G. P.; Gonzalez, R. R.; Hernandez, C. T. Tetrahedron 1996, 52, 4817.

(3) Ryu, I.; Kreimerman, S.; Araki, F.; Nishitani, S.; Oderatoshi, Y.; Minakata, S.; Komatsu, M. J. Am. Chem. Soc. 2002, 124, 3812. 


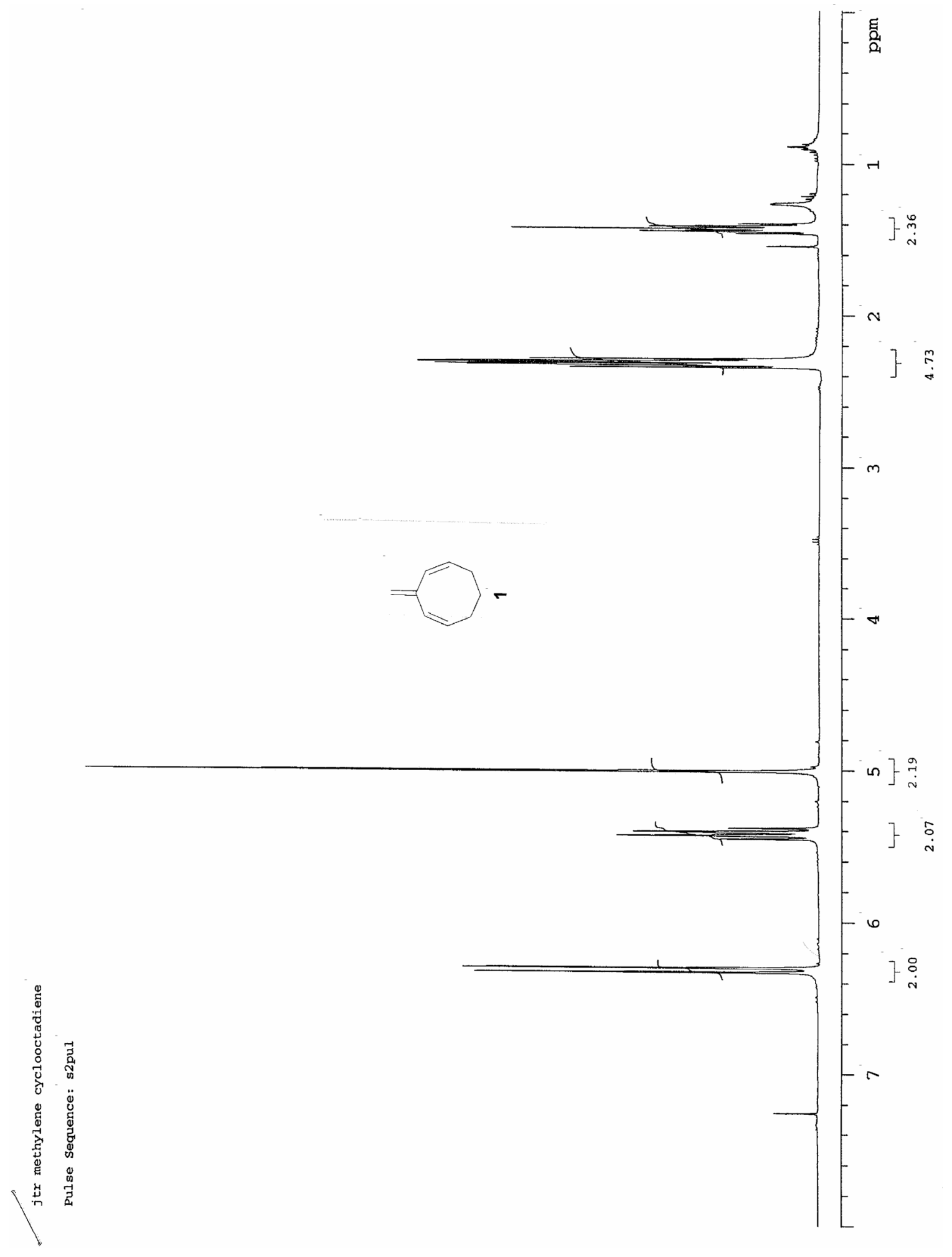




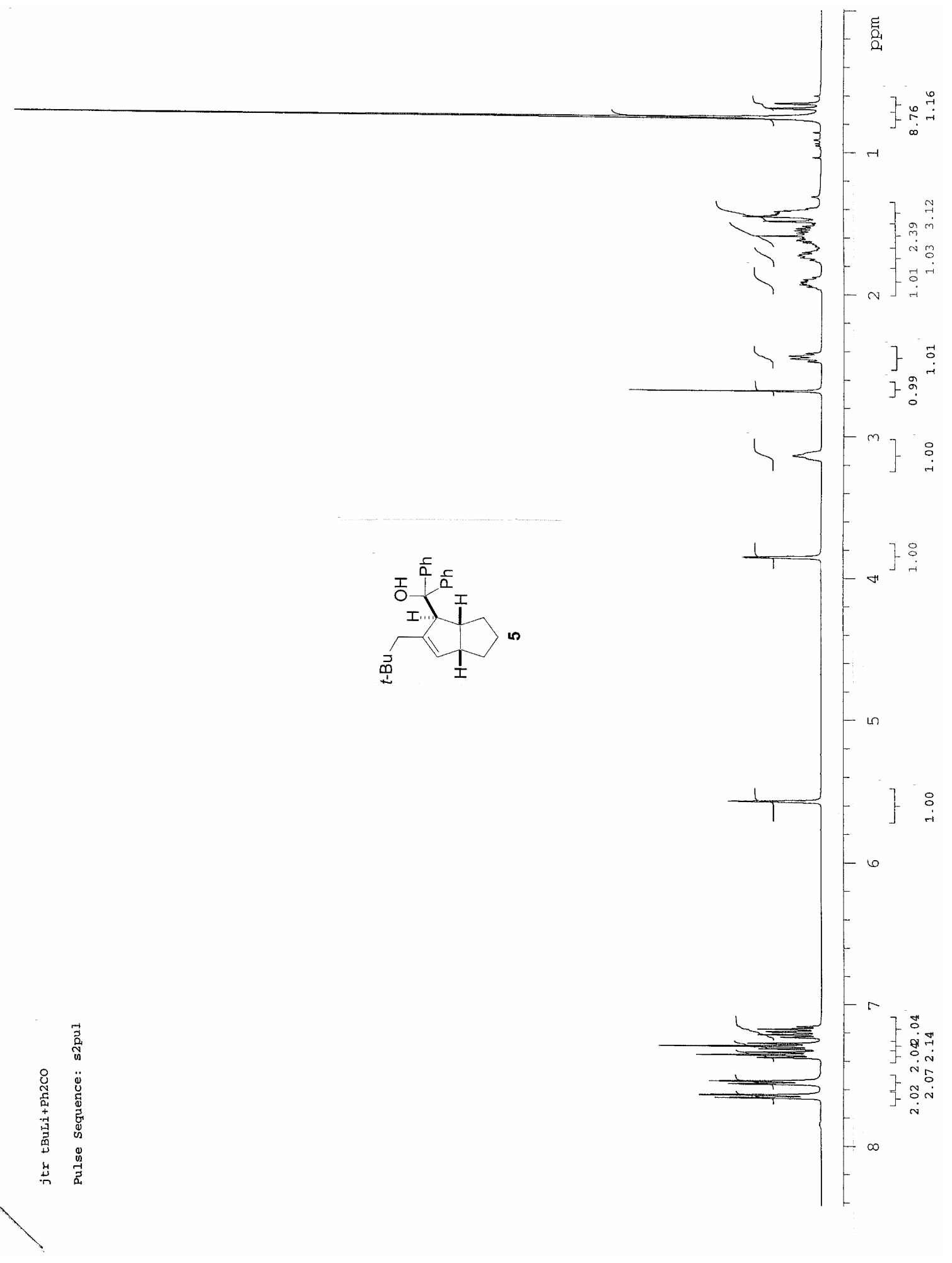




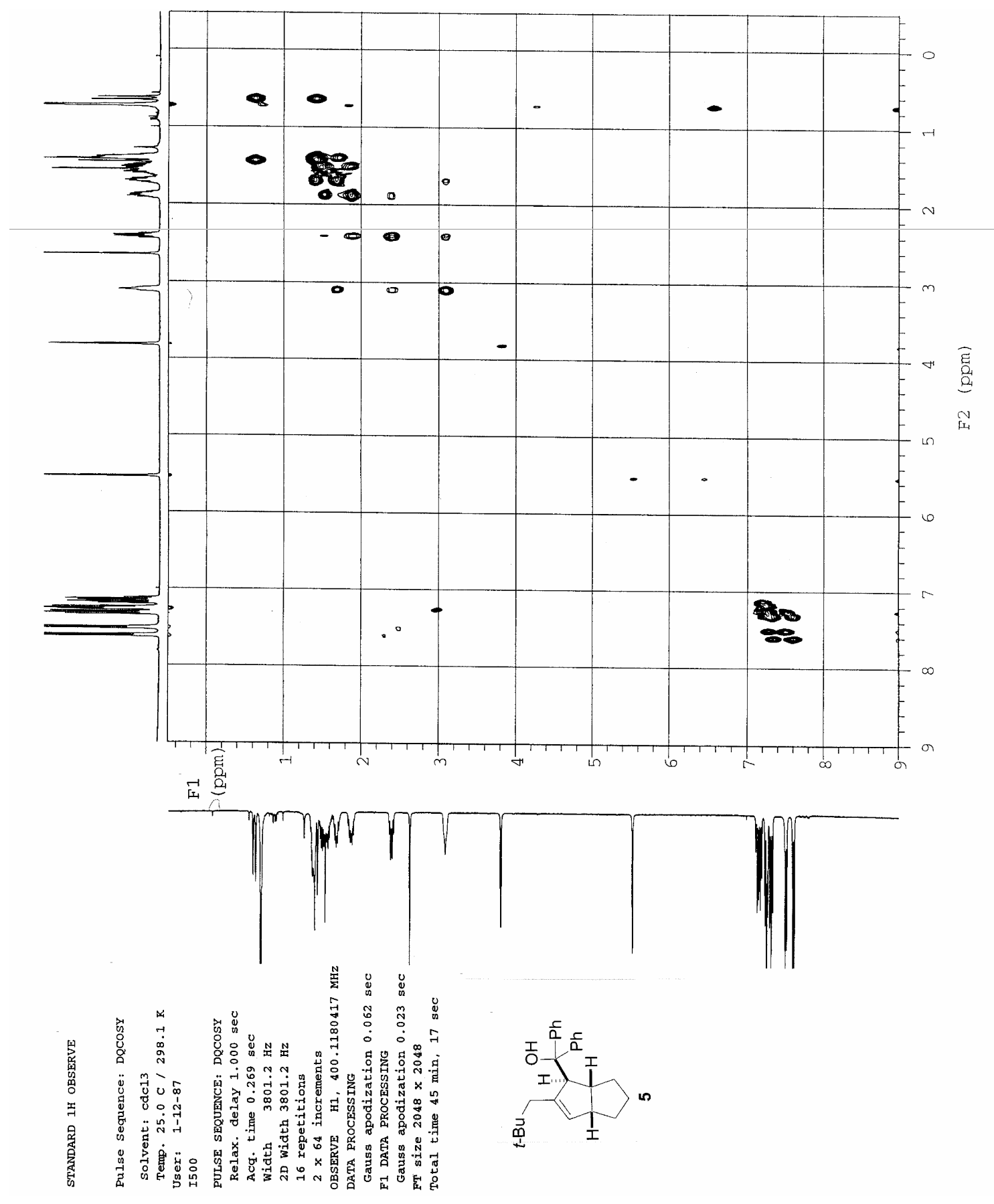




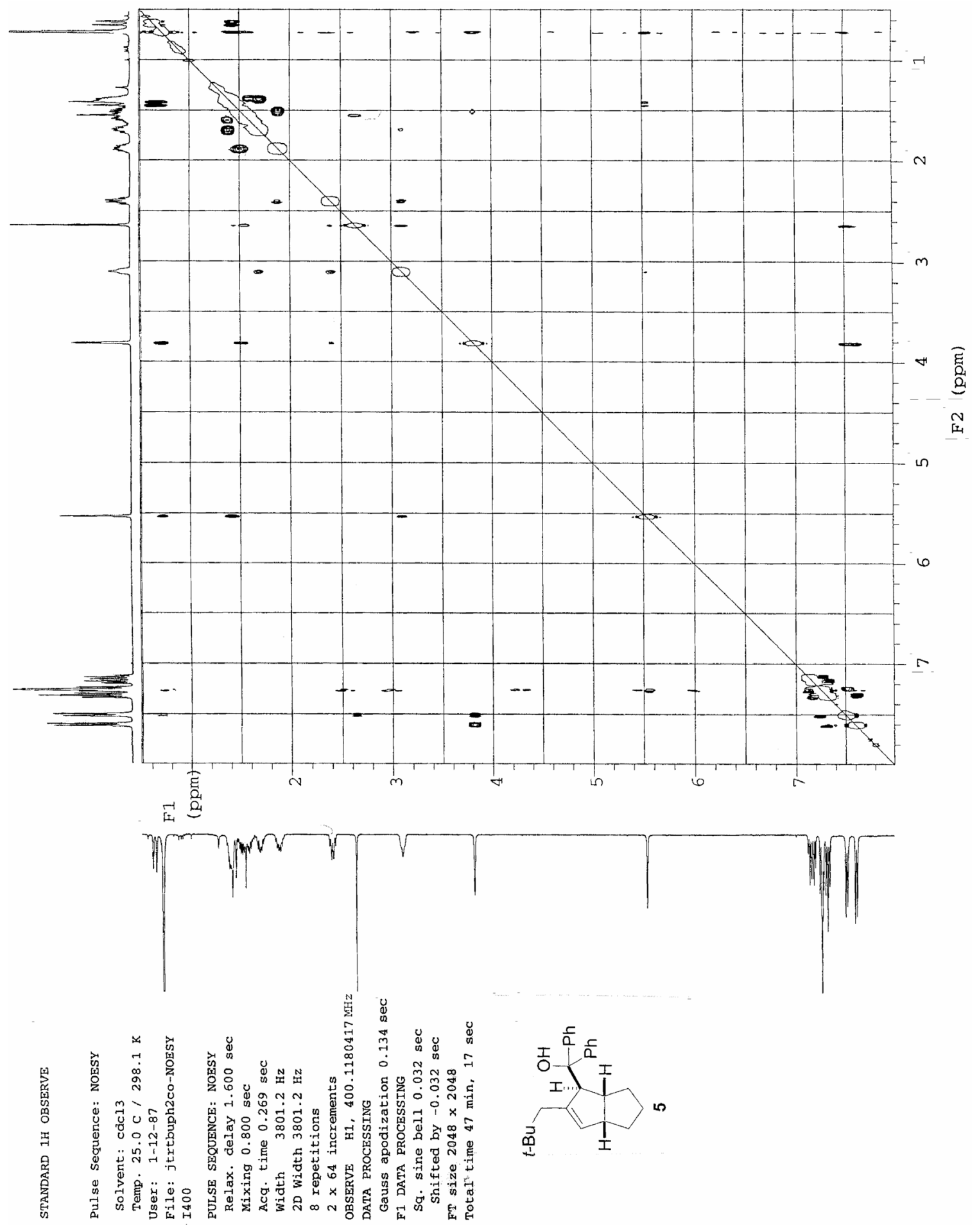




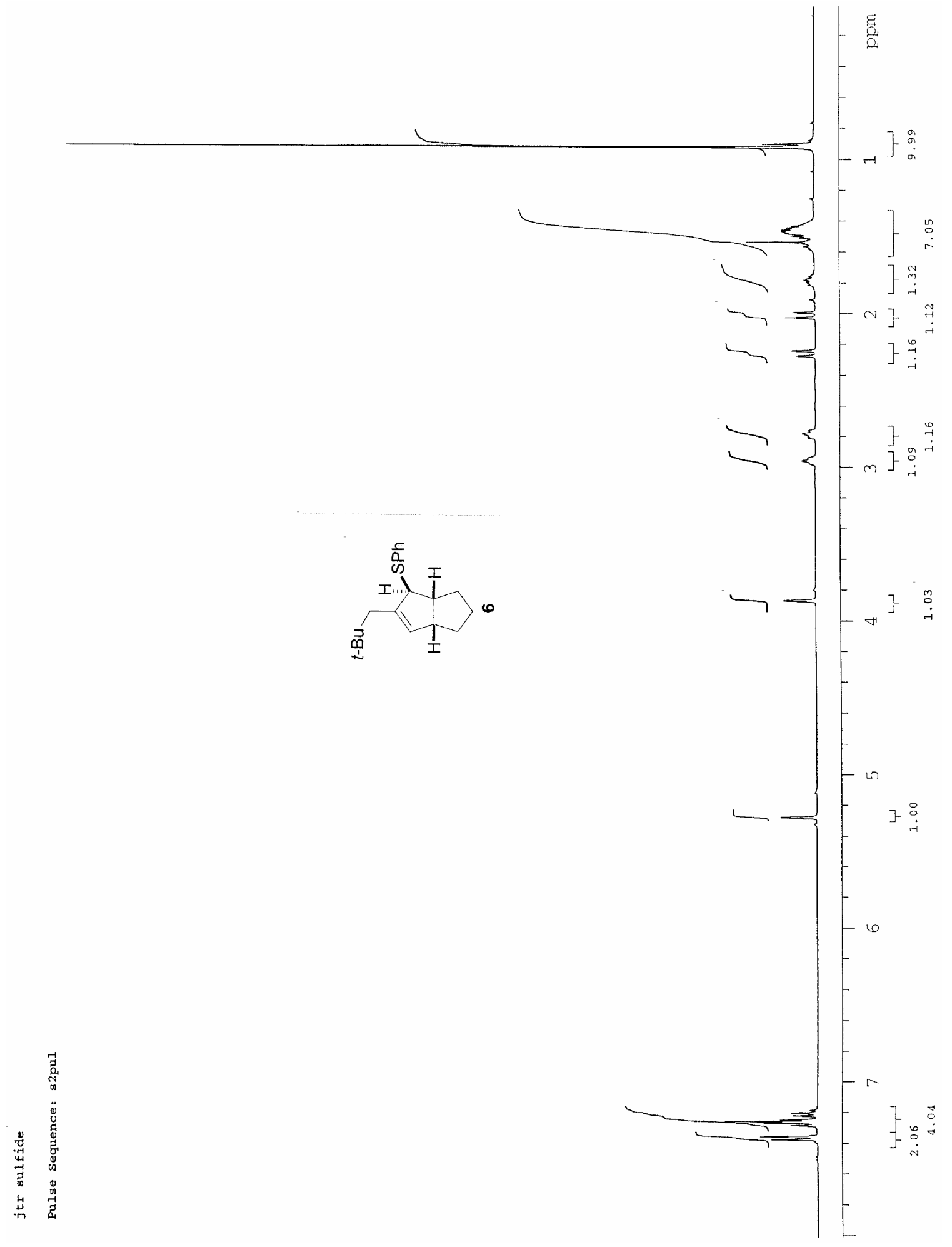




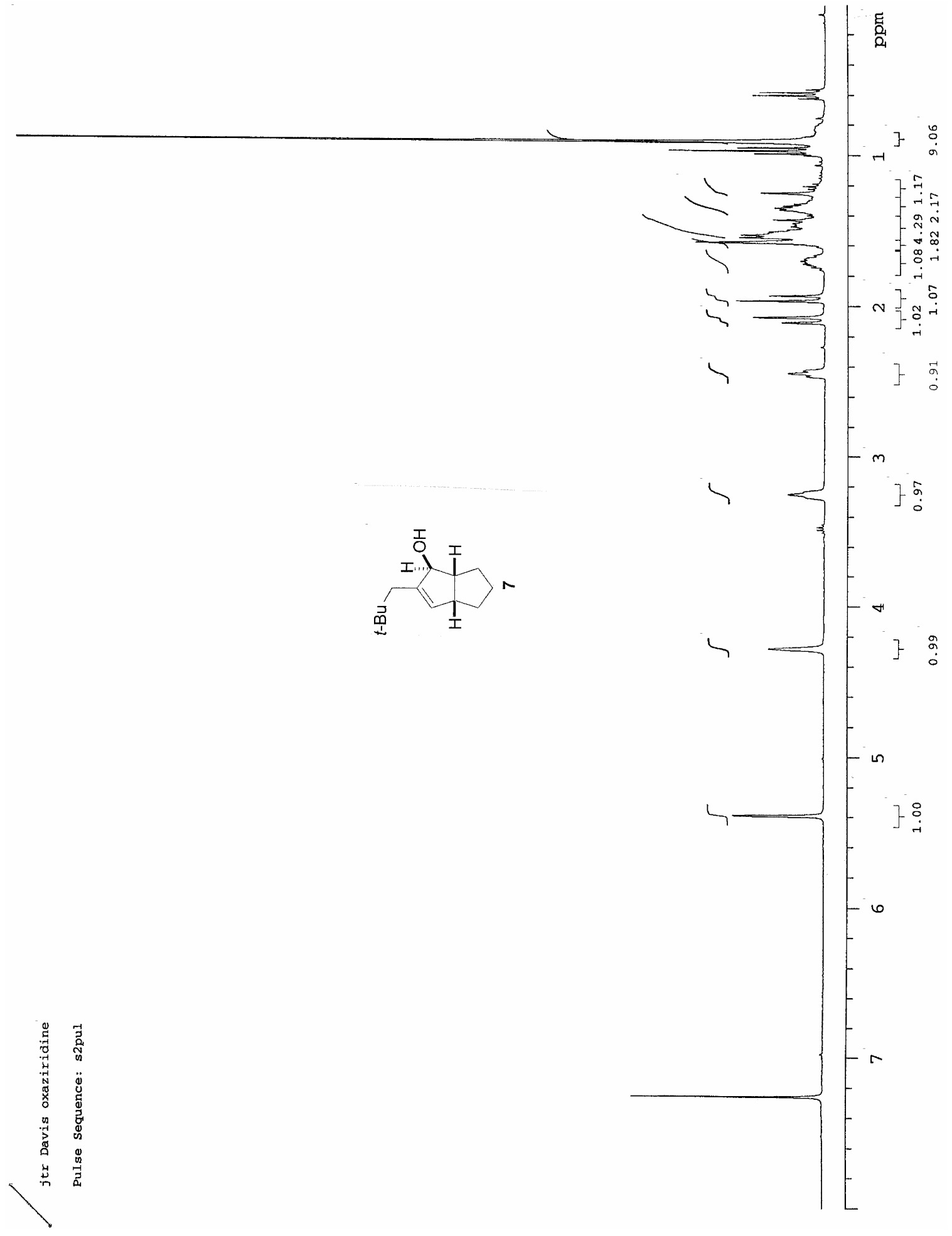




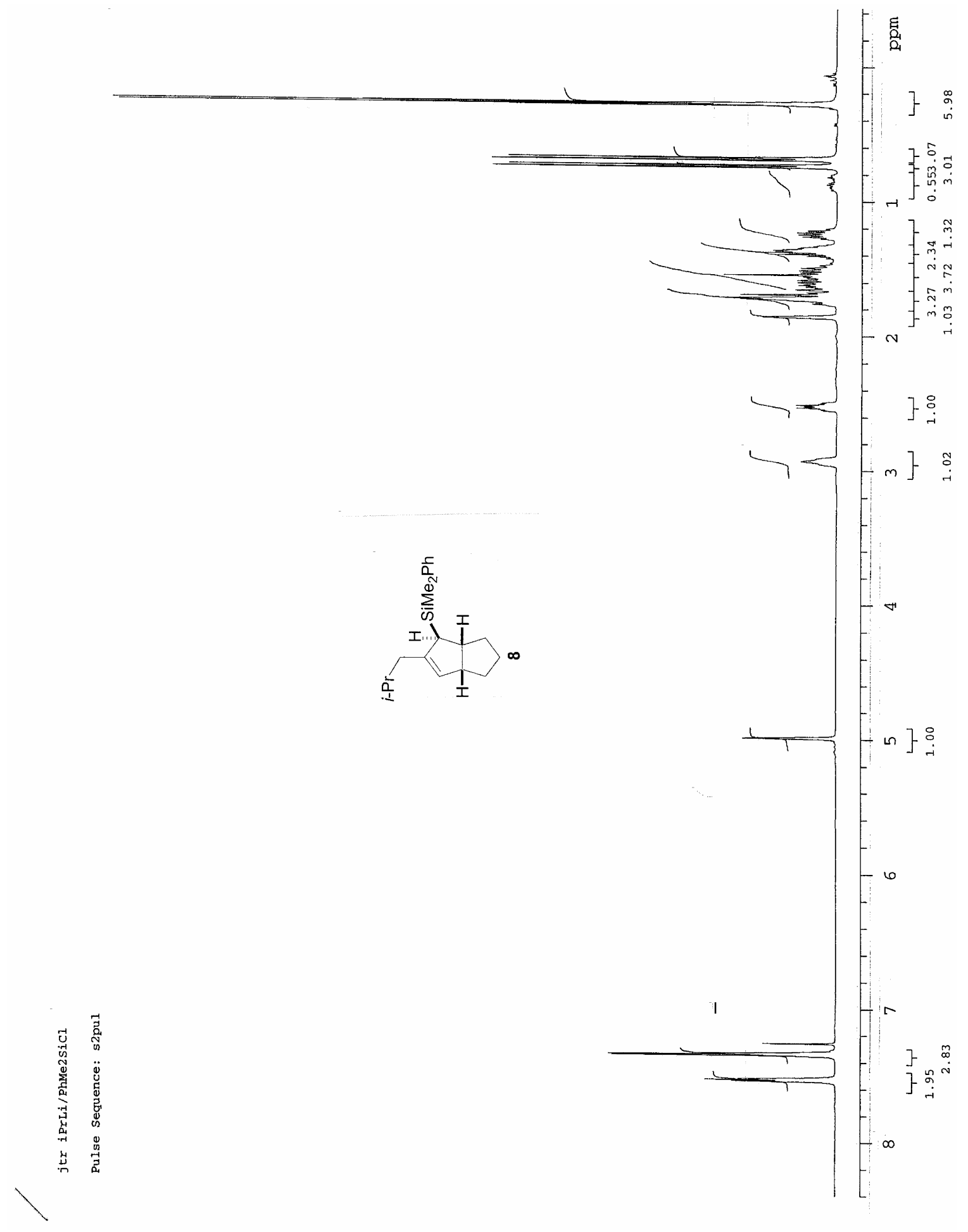




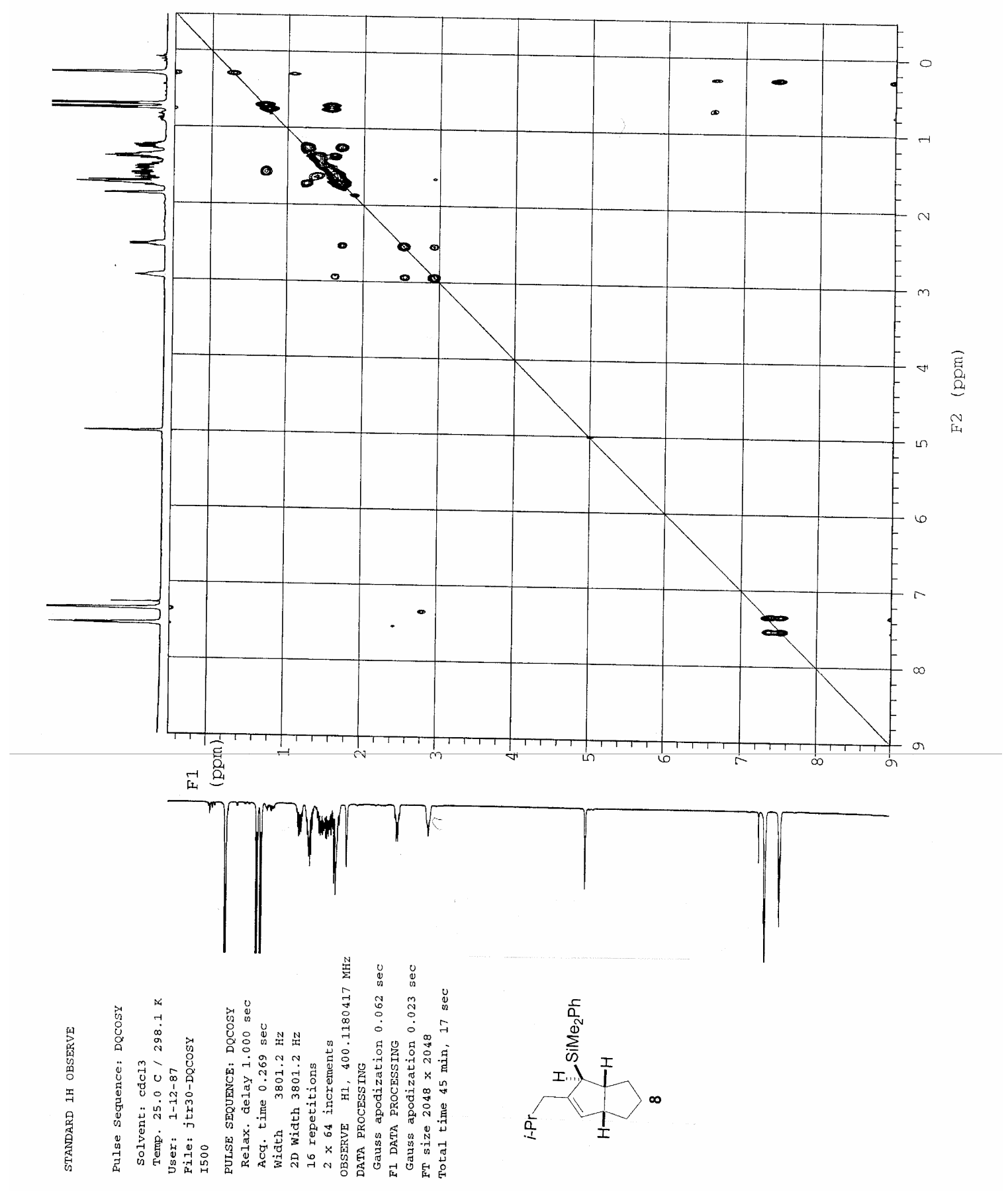




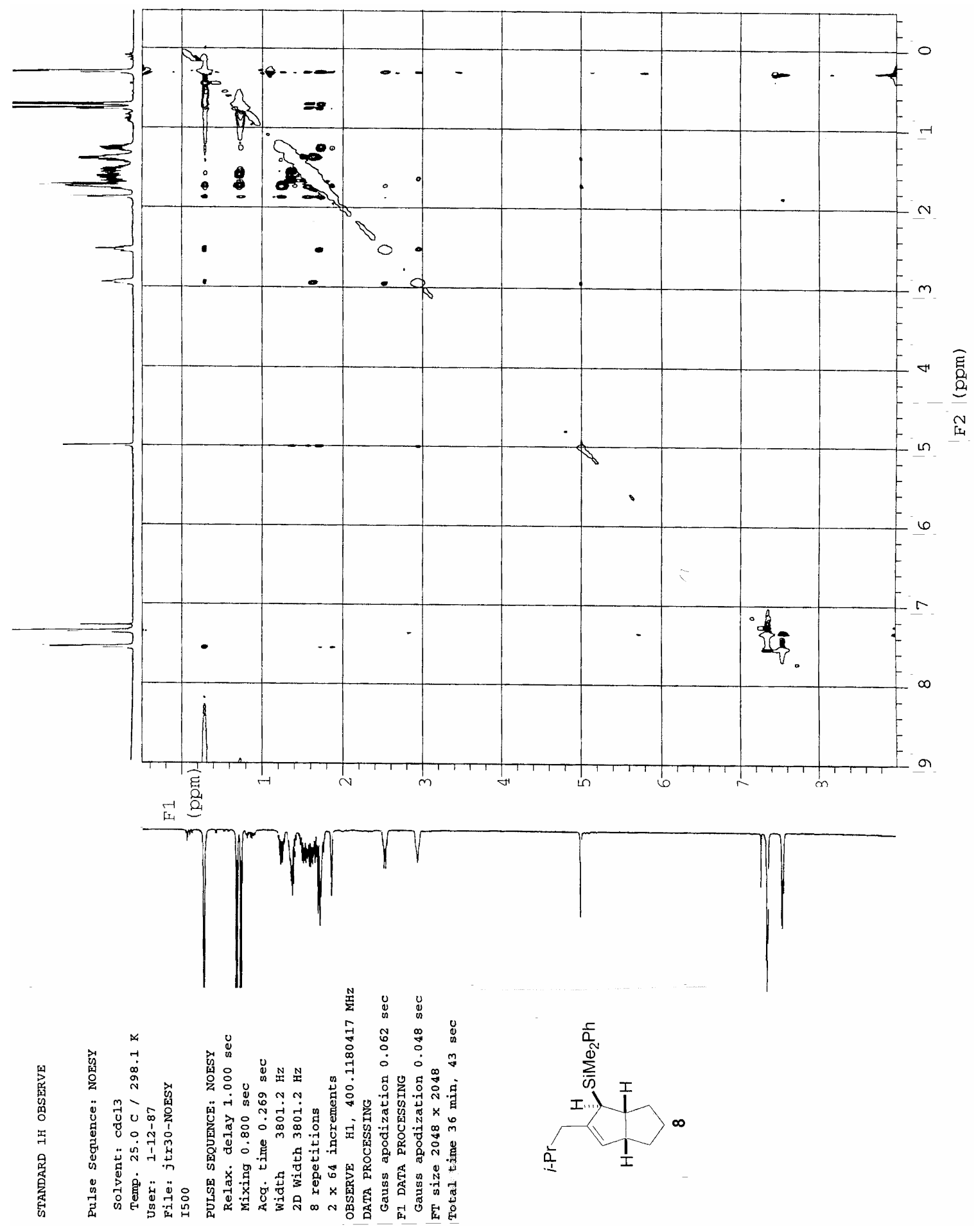




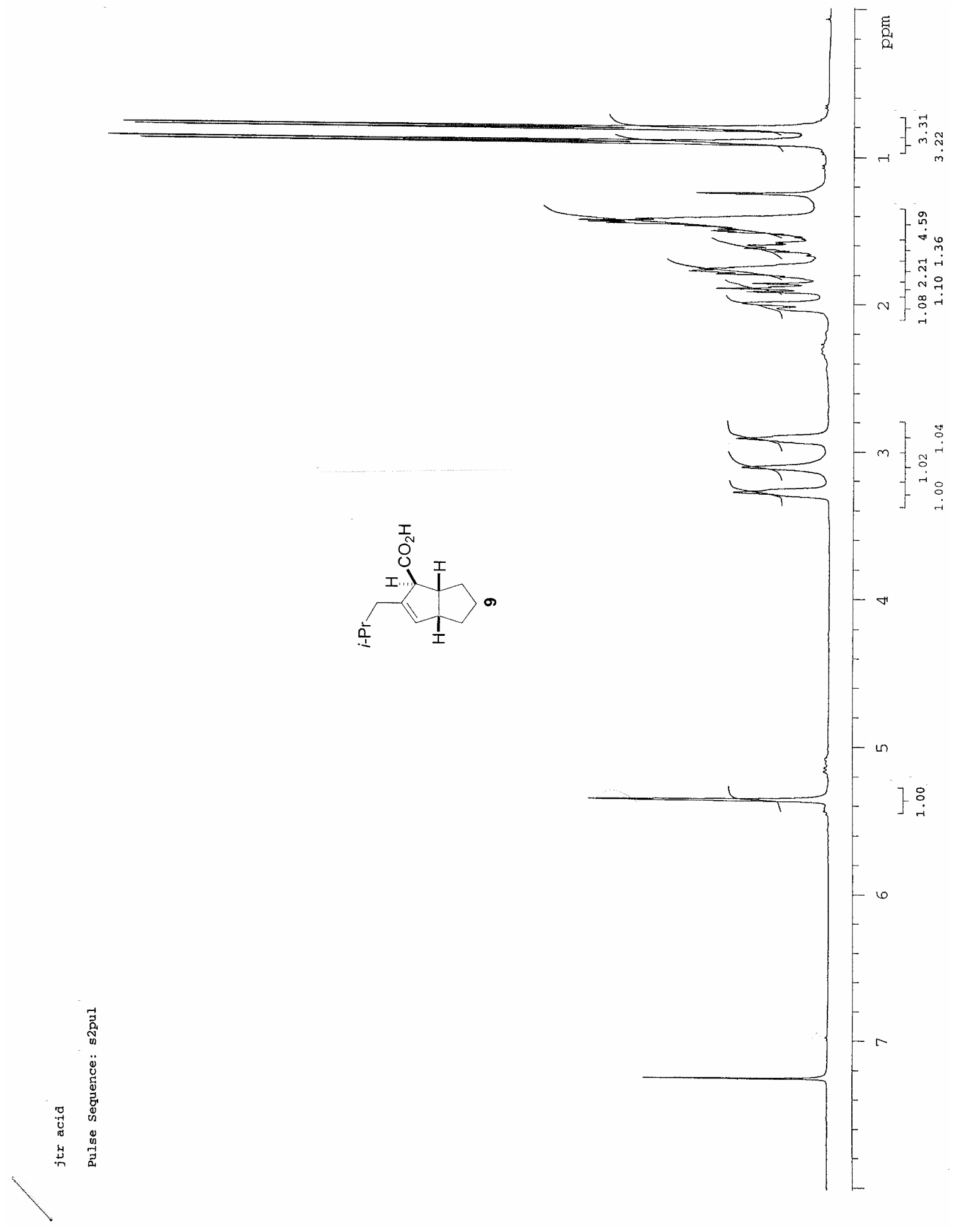




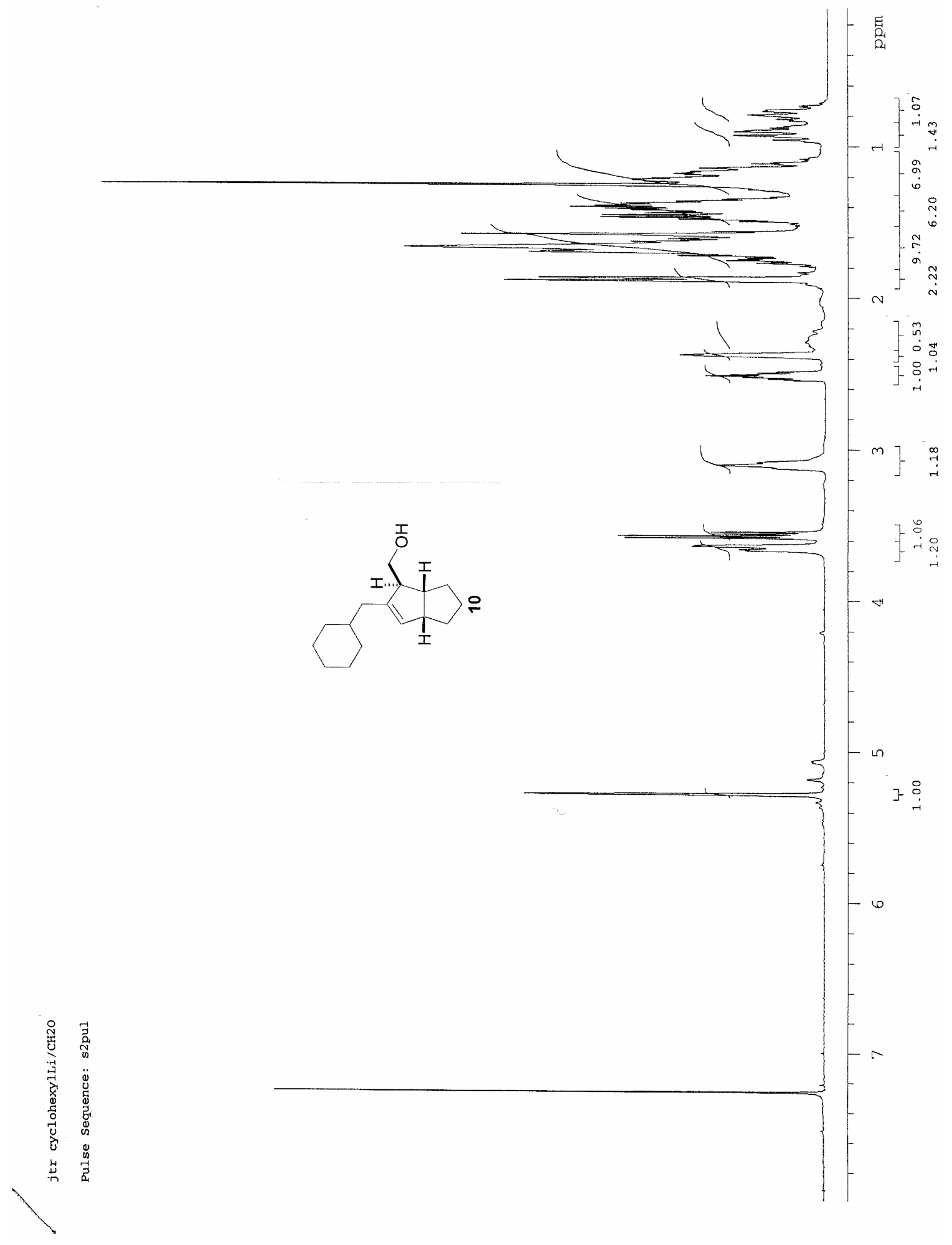




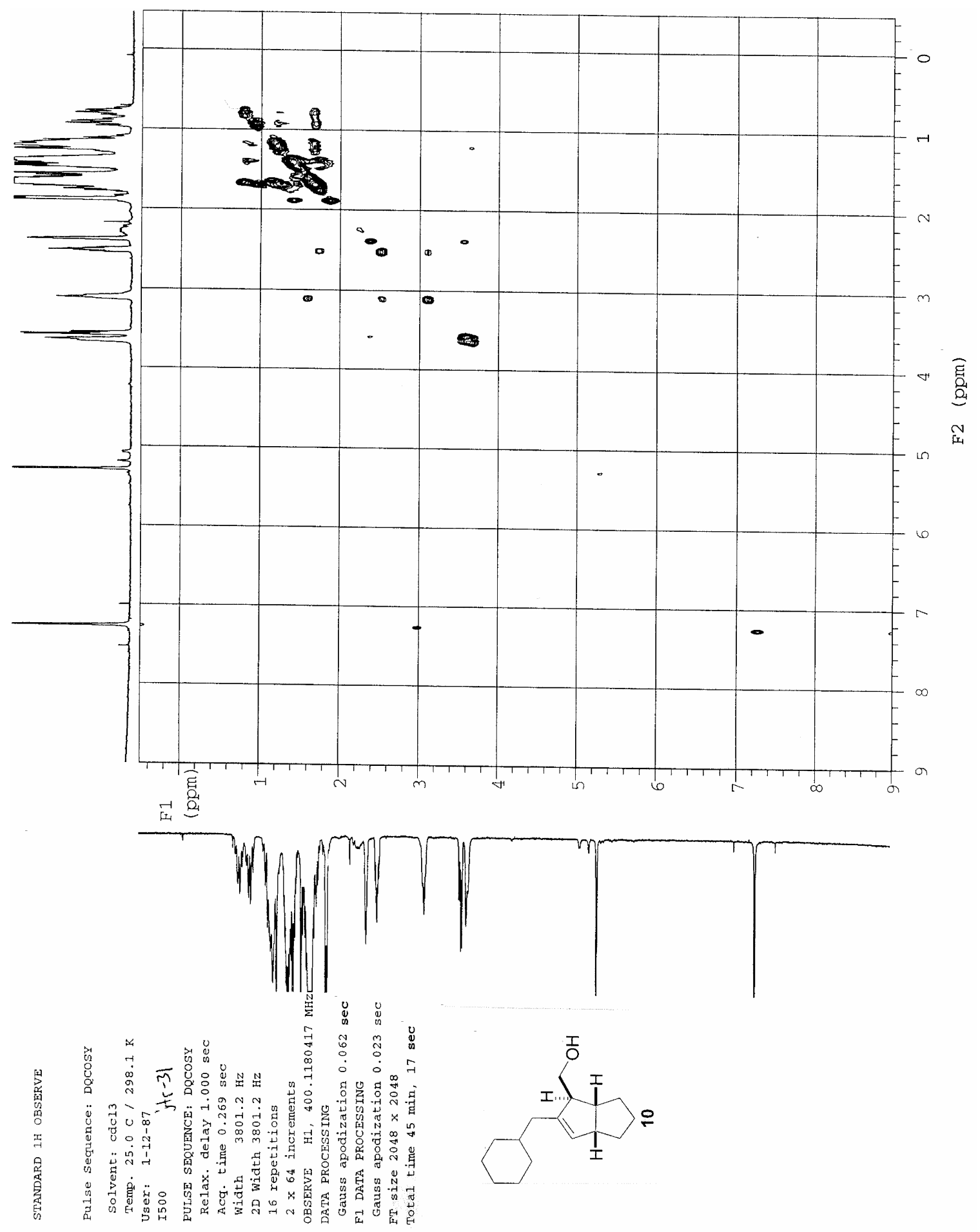




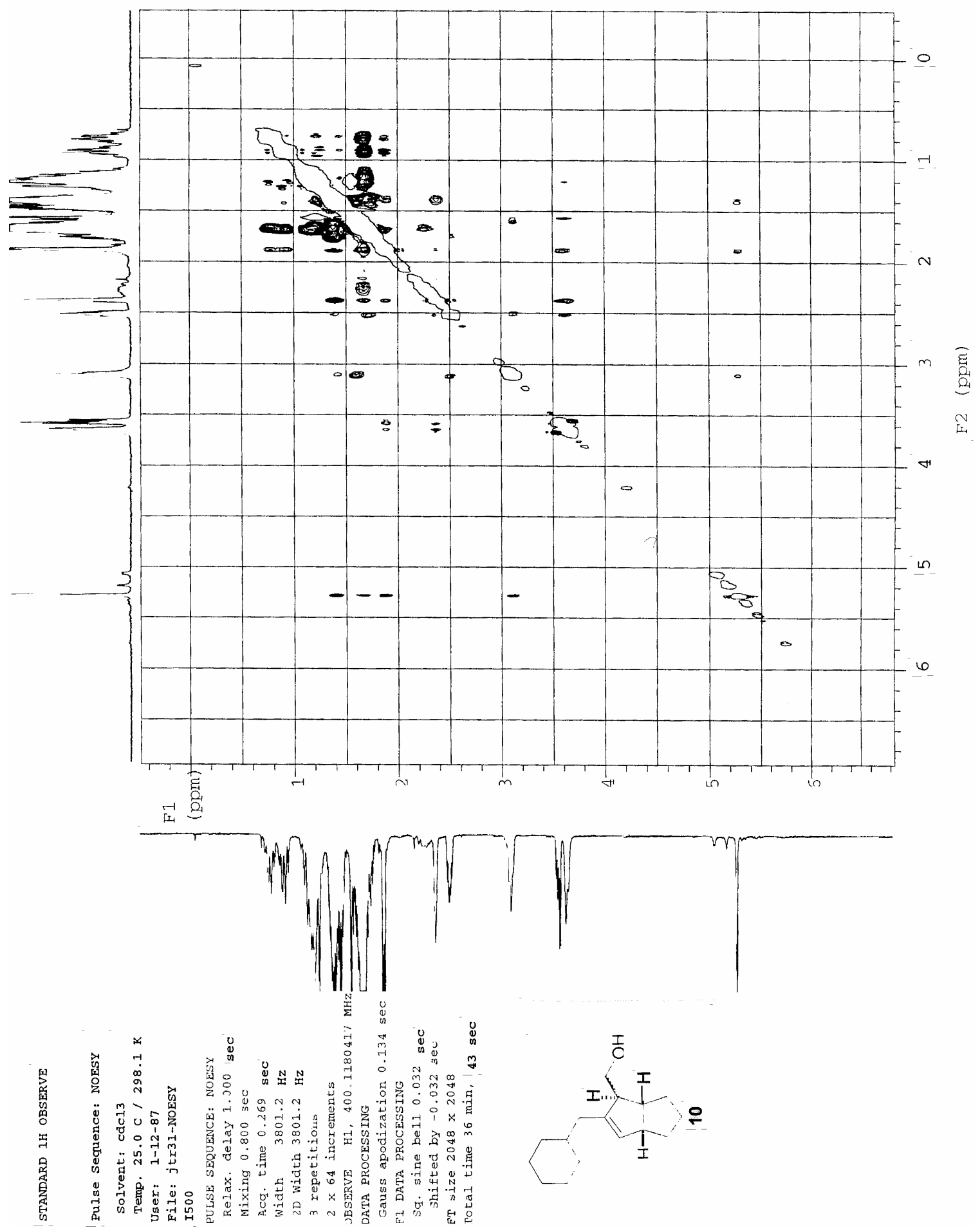




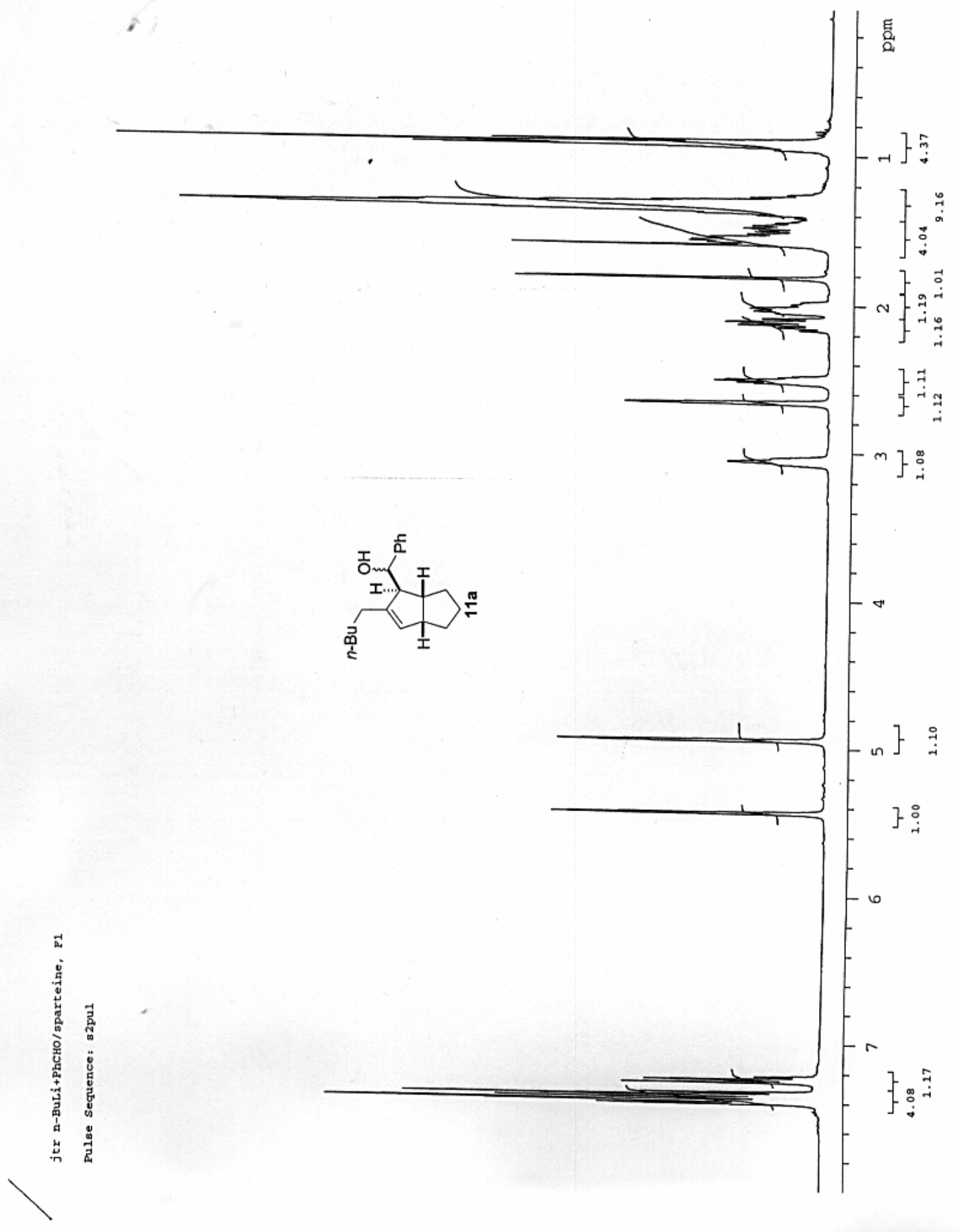




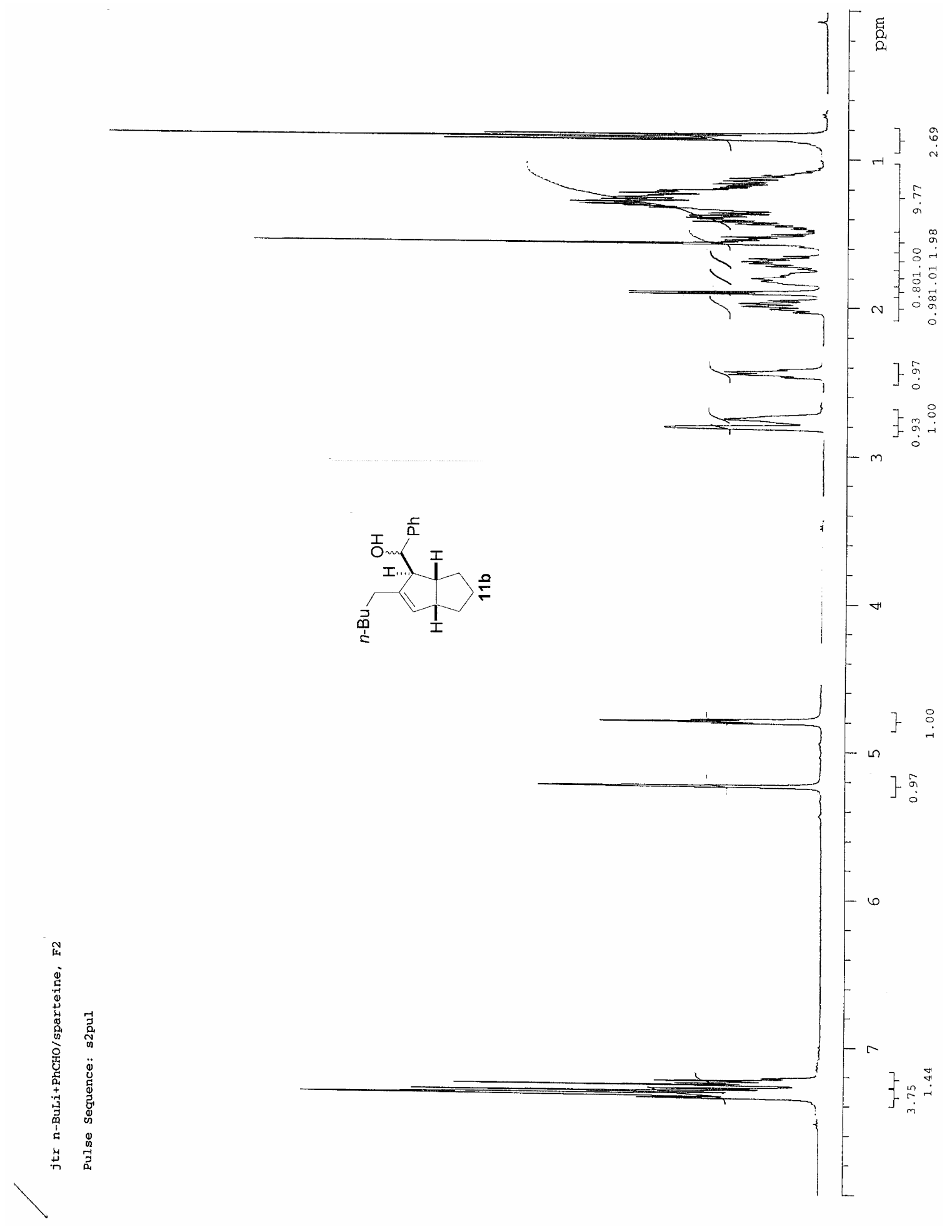




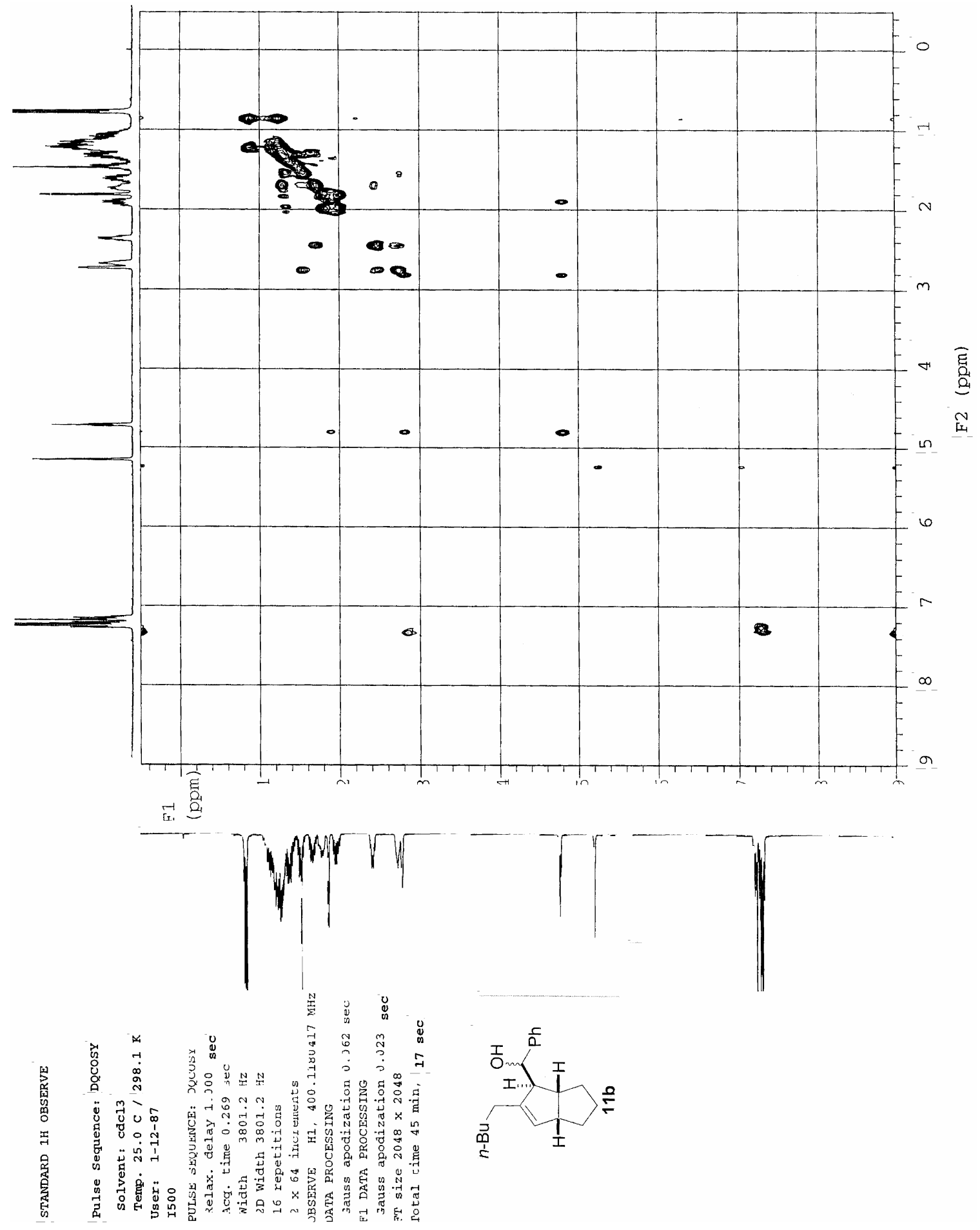




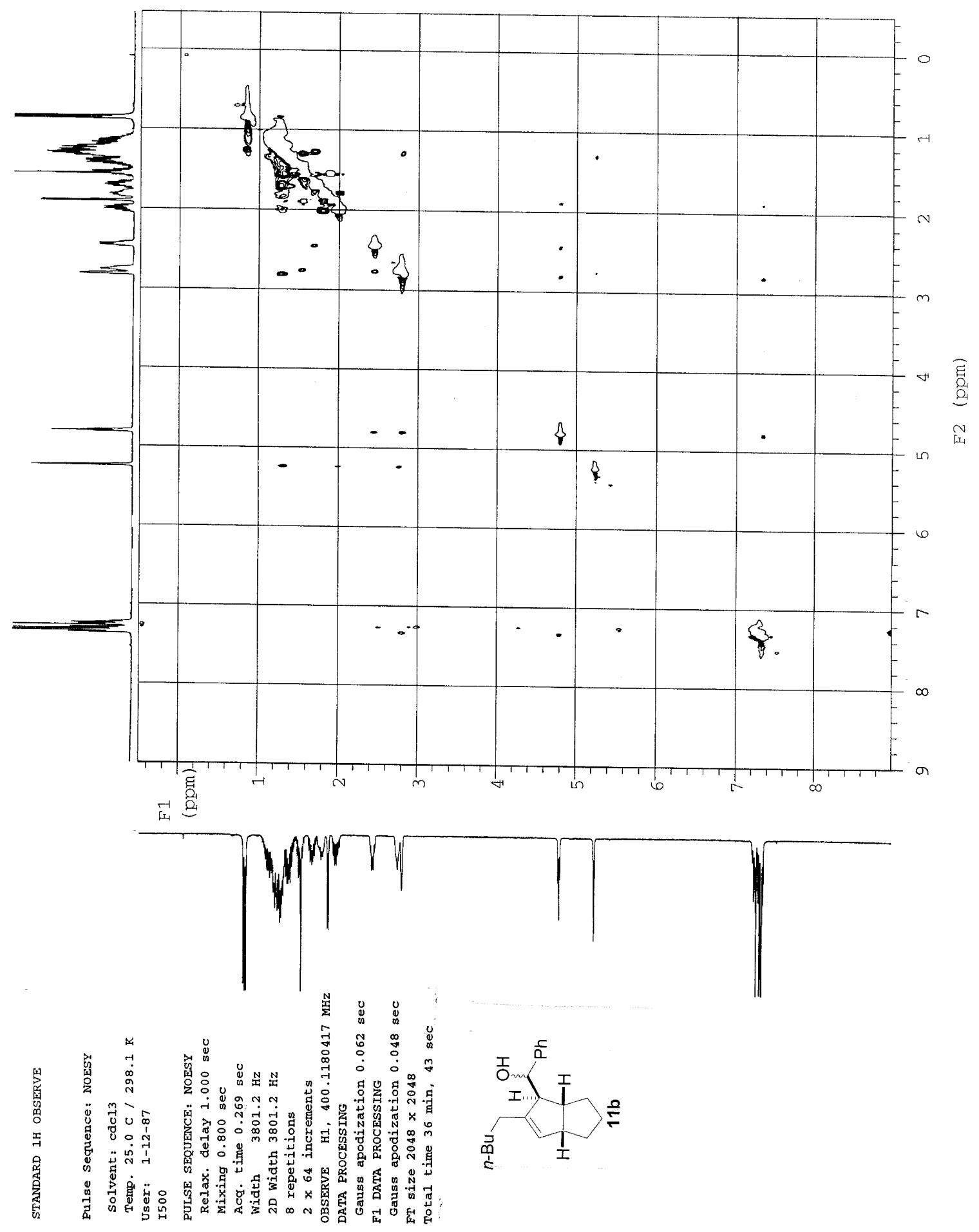




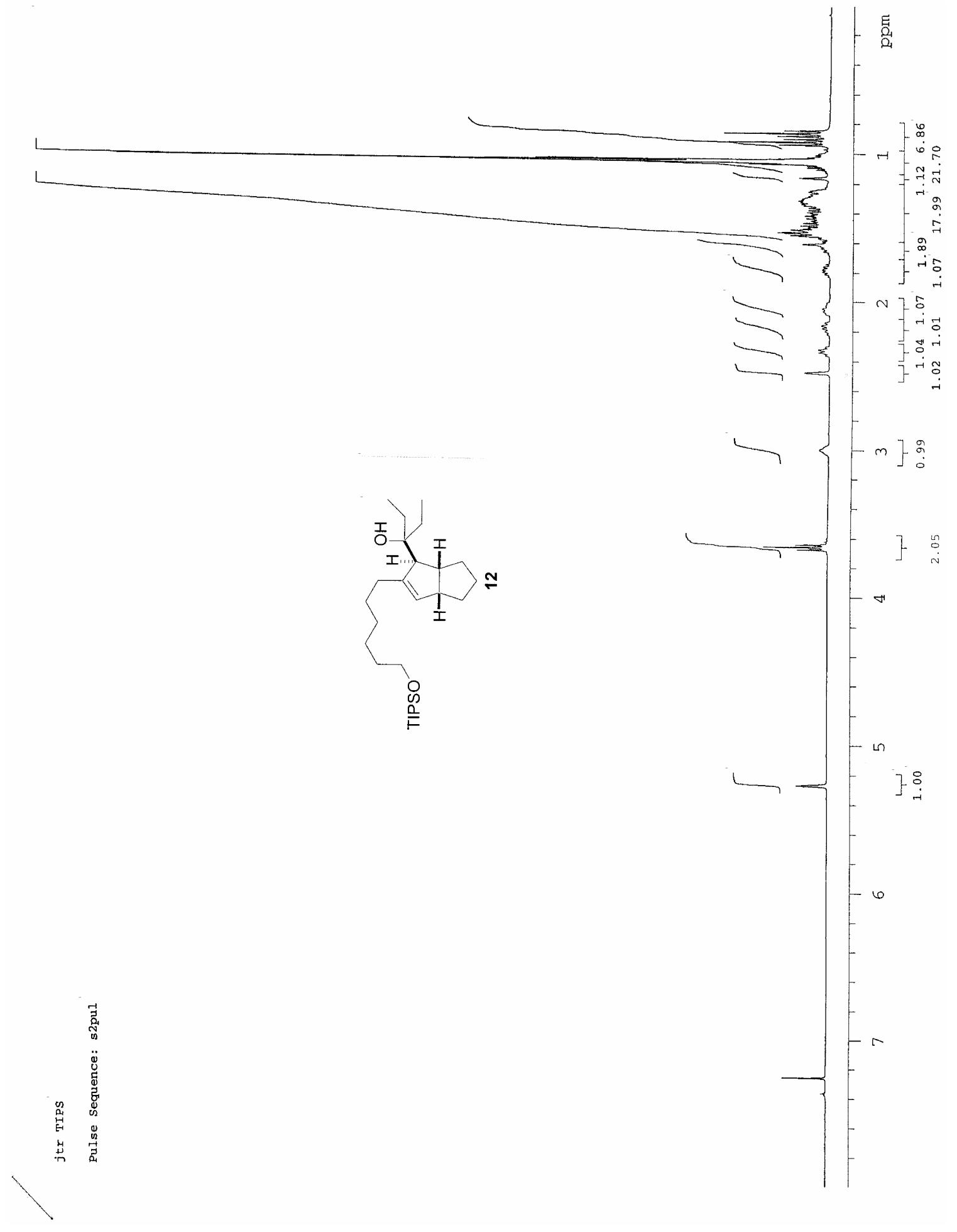




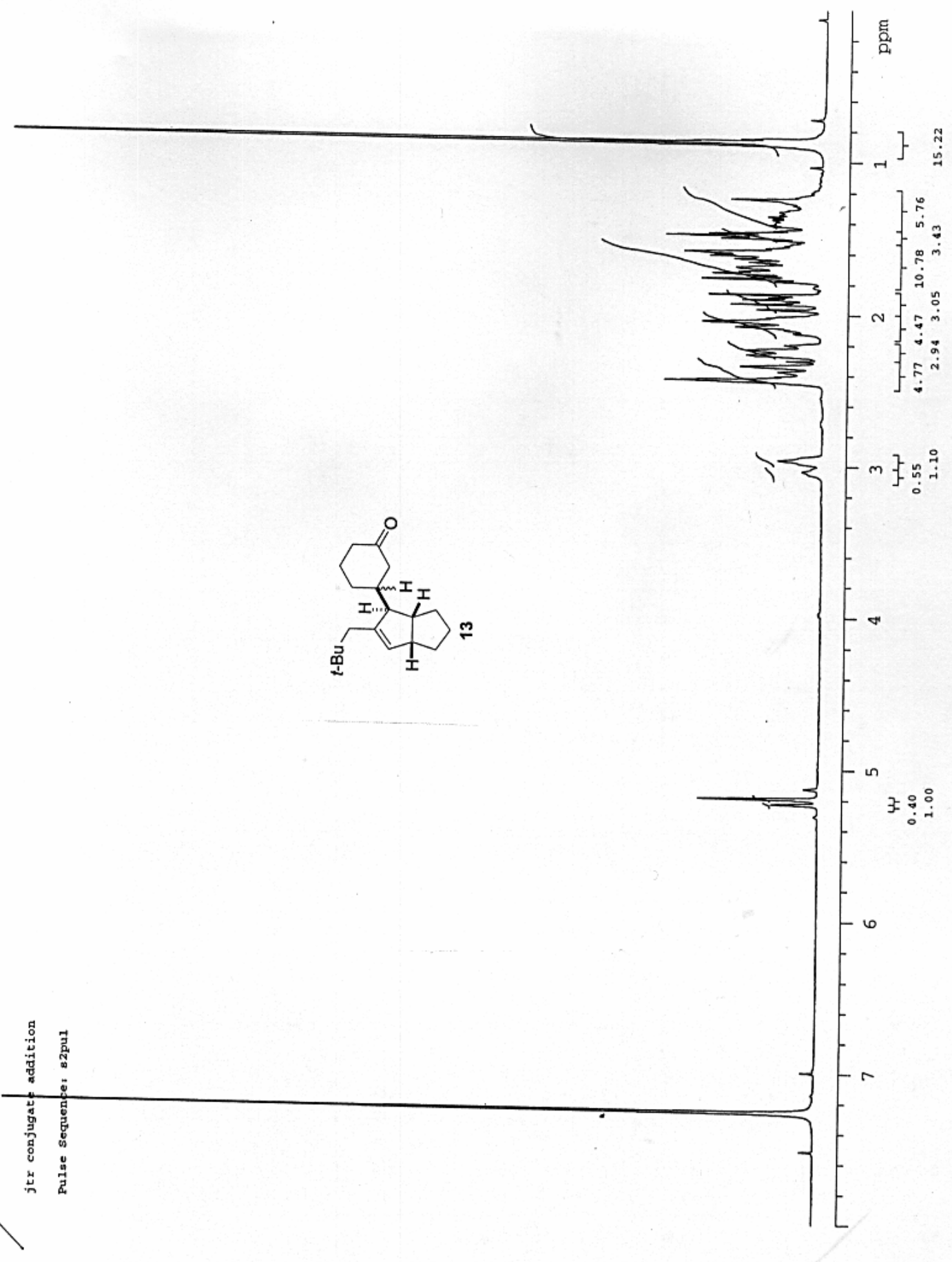




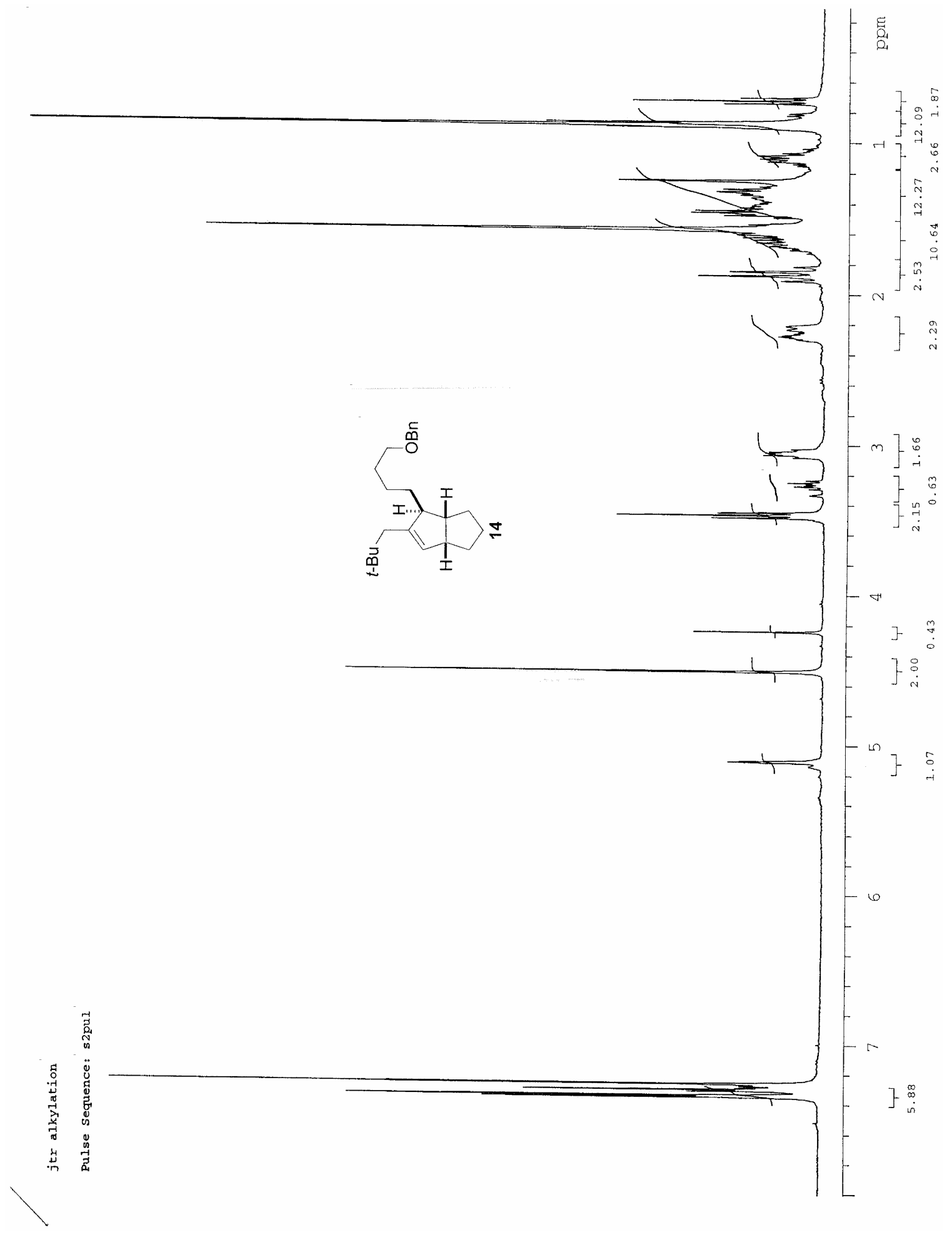




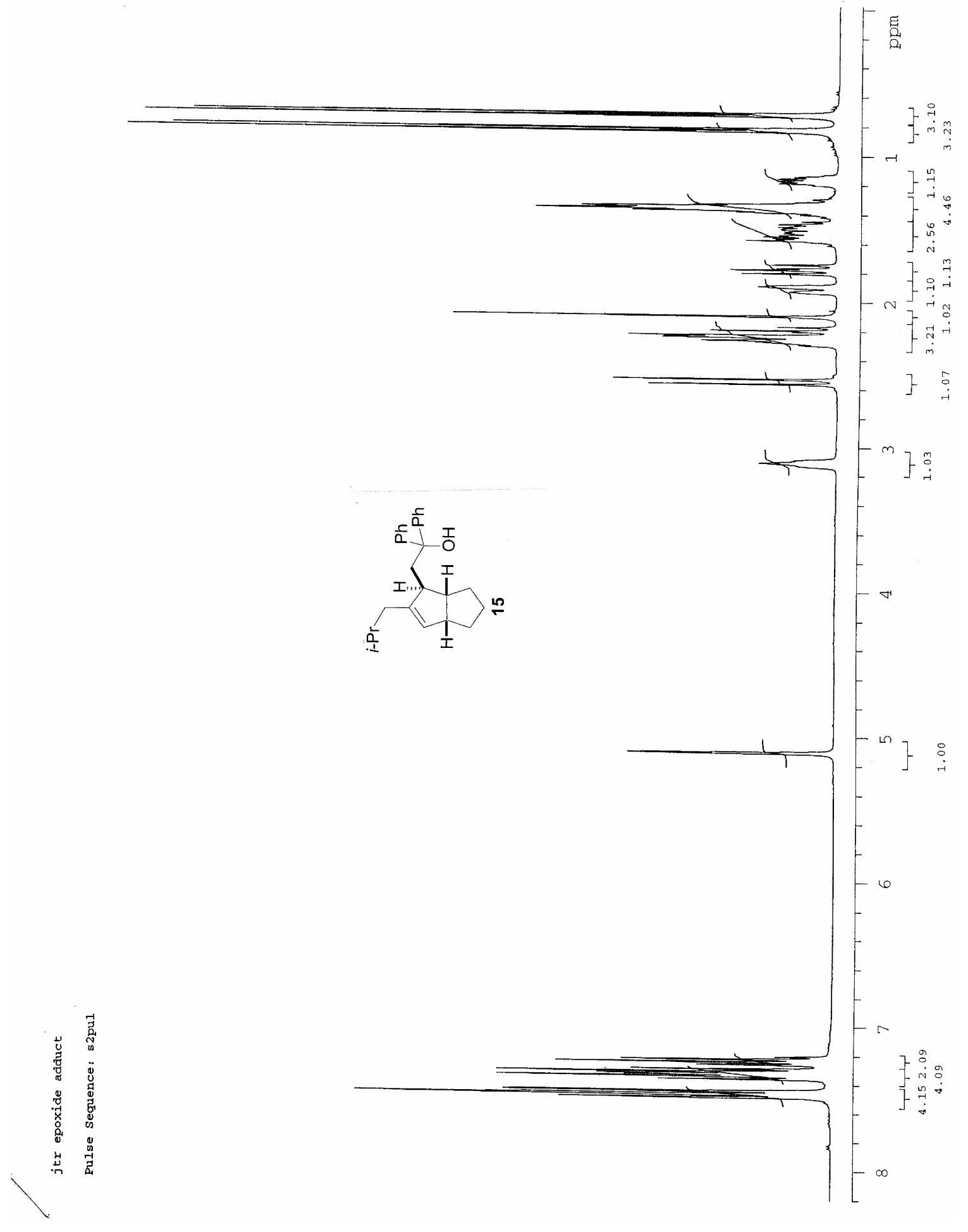




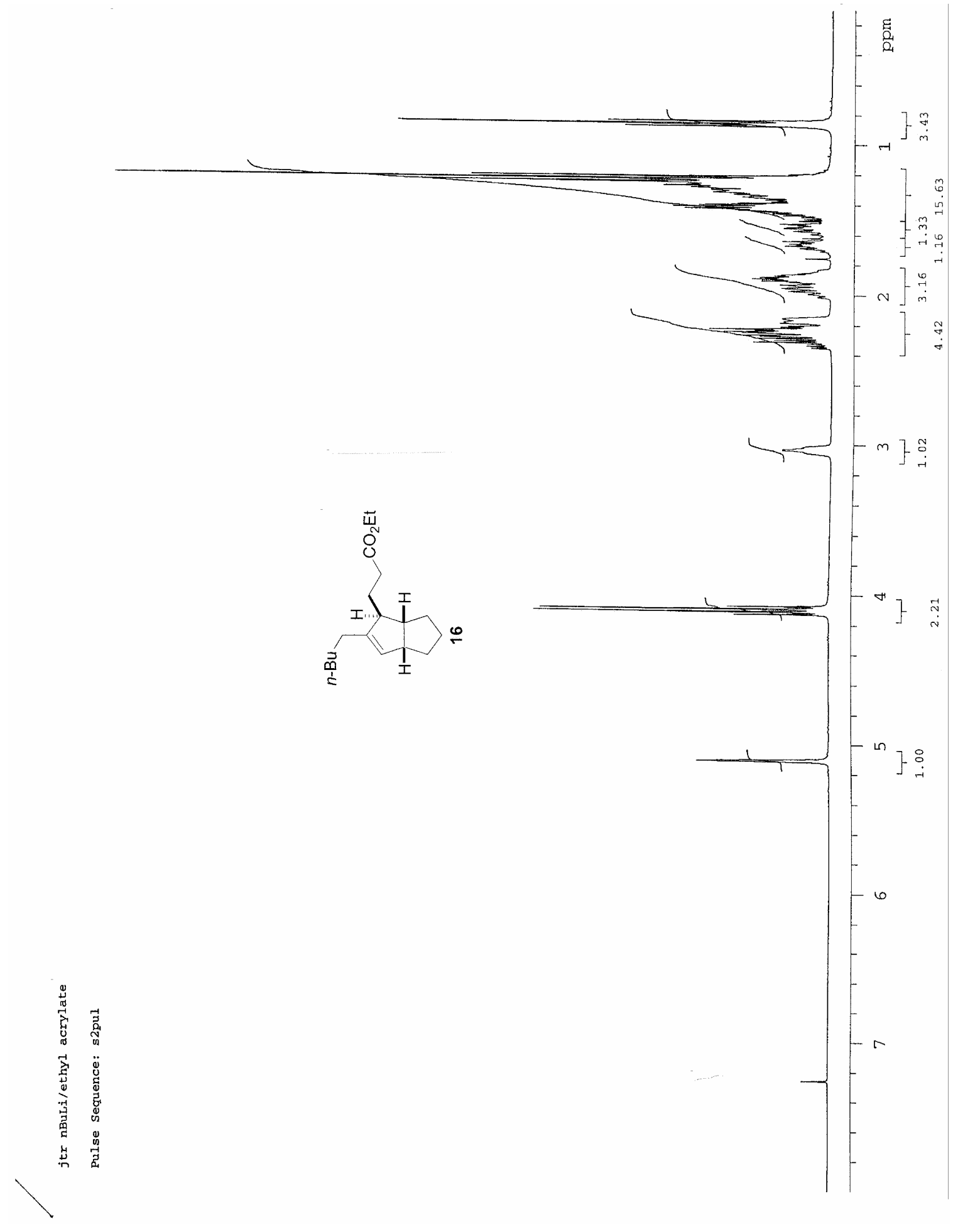




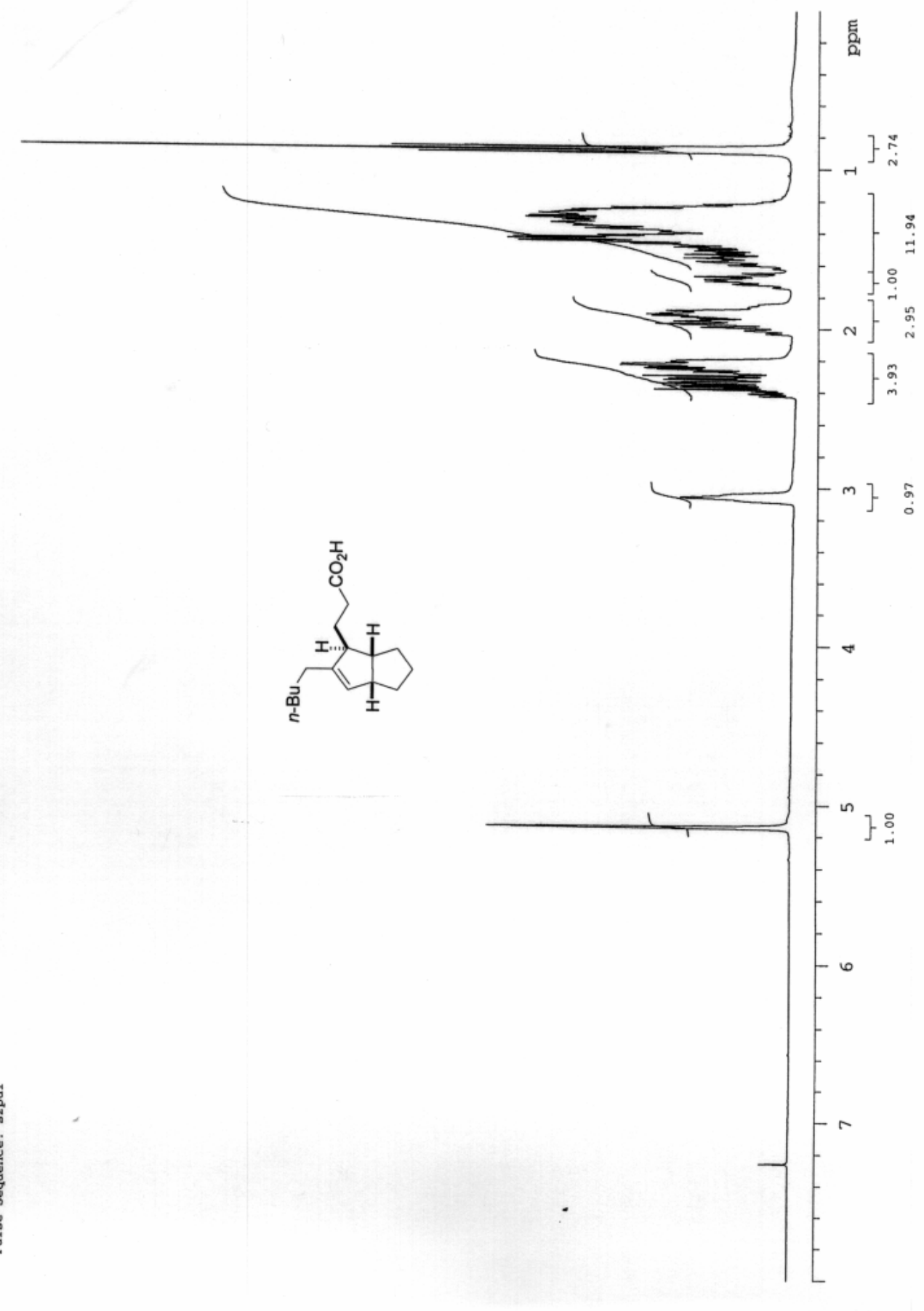




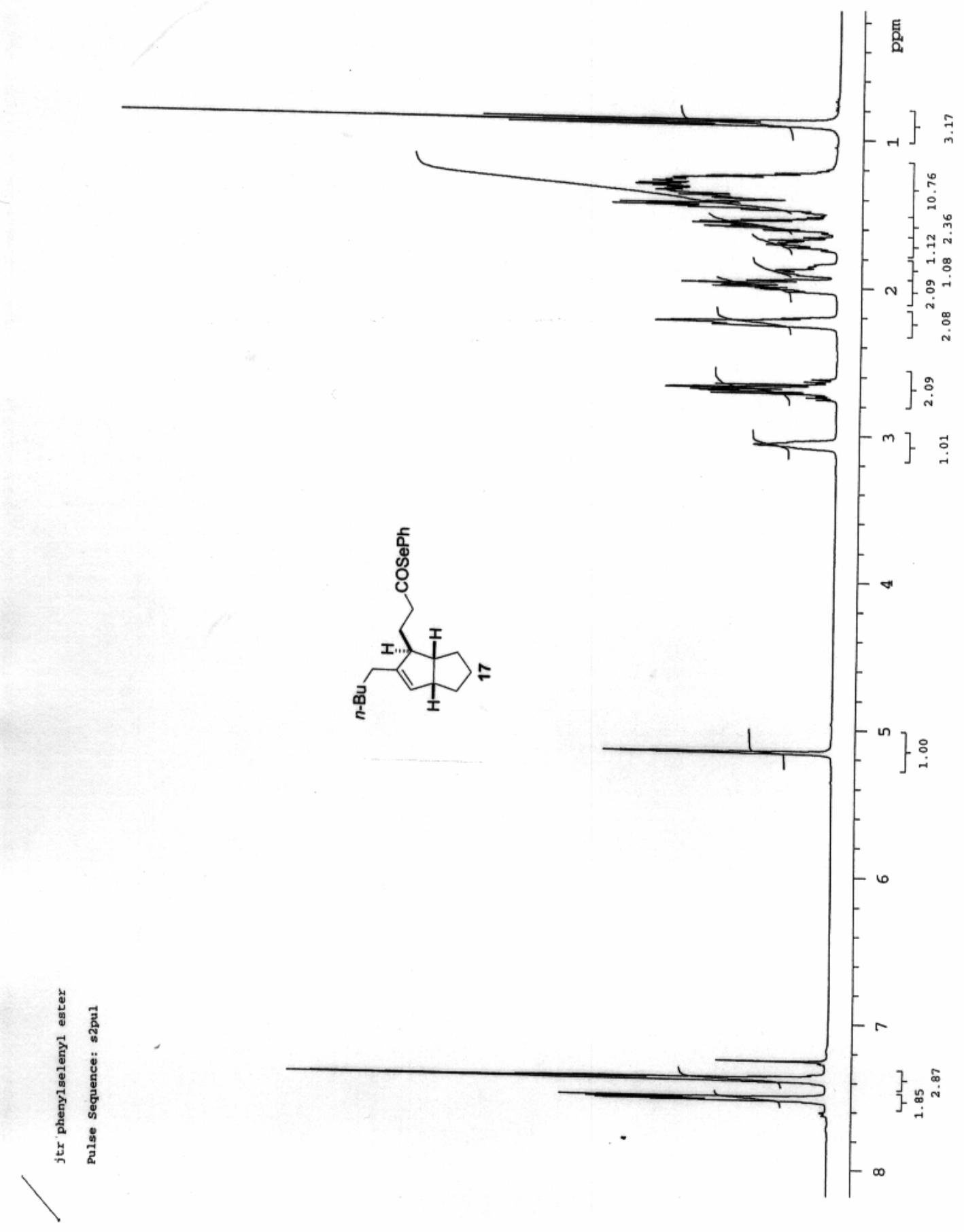




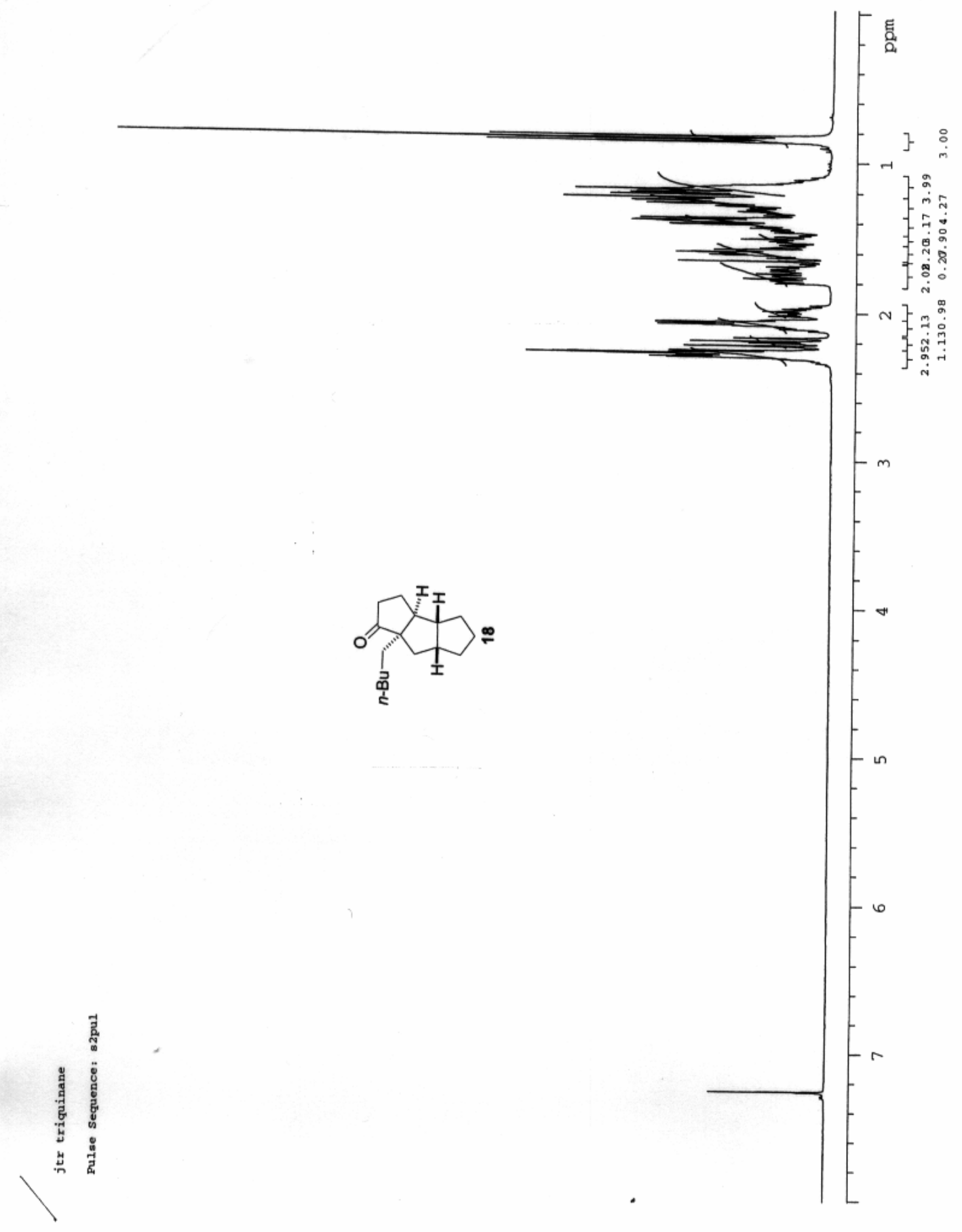




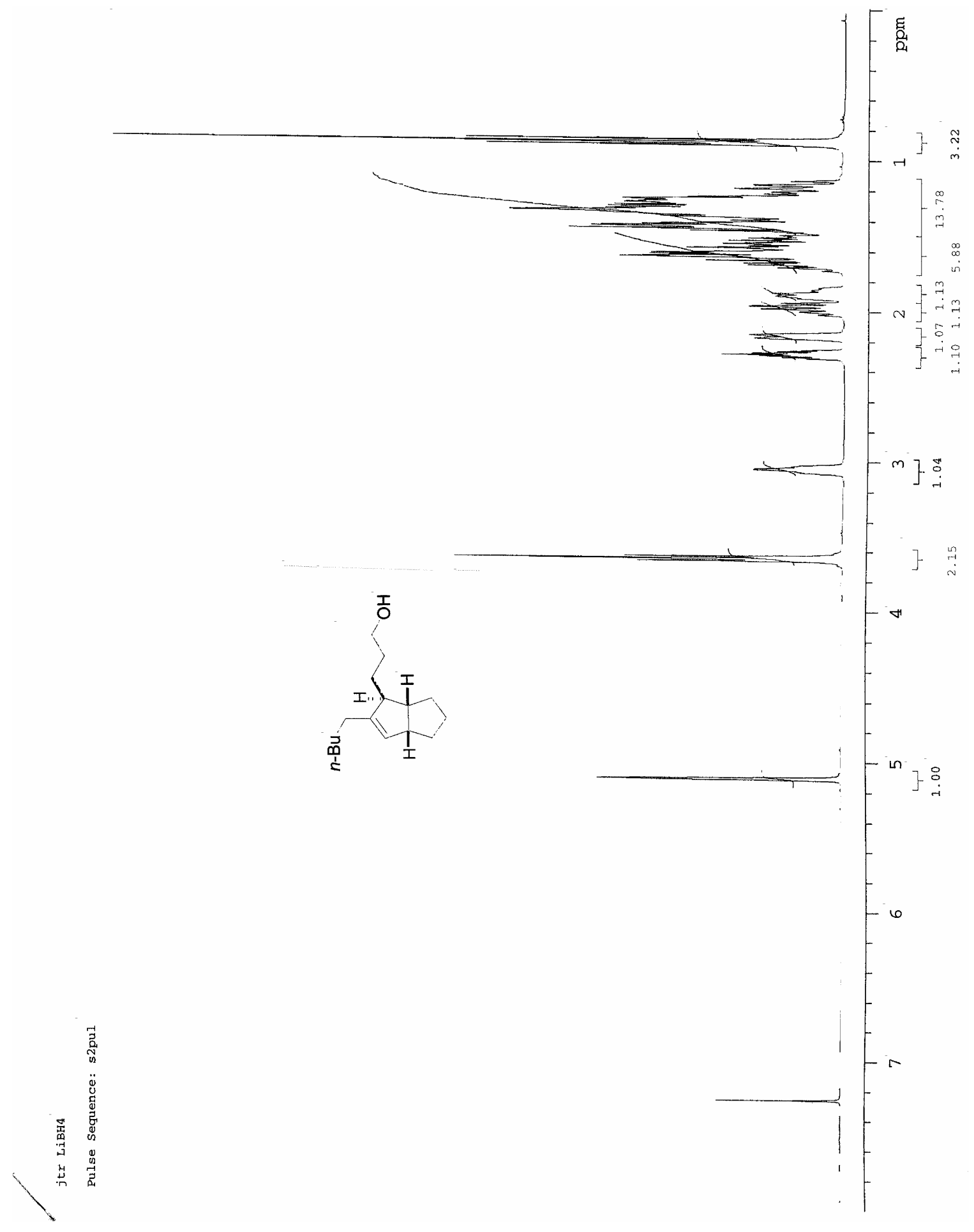




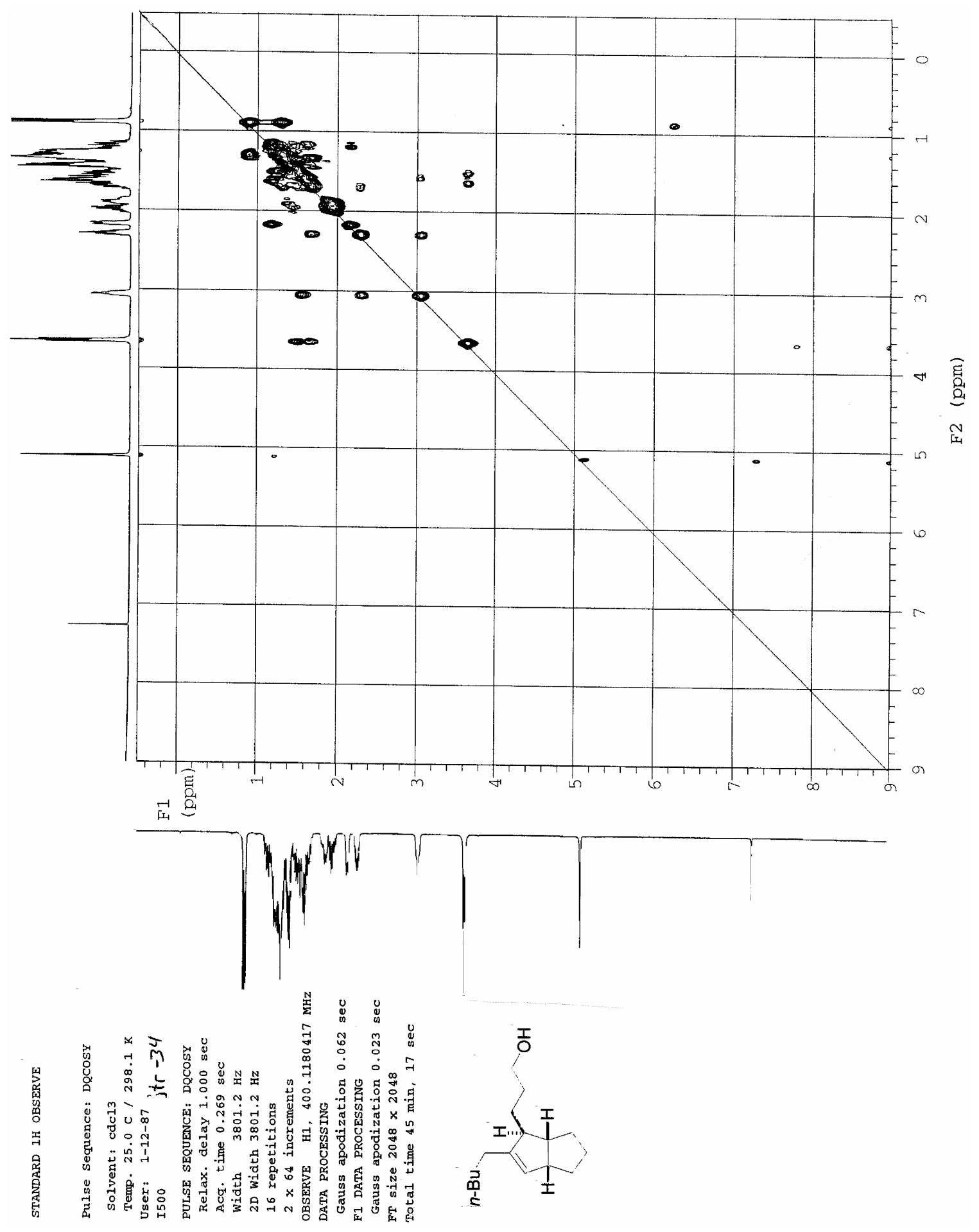




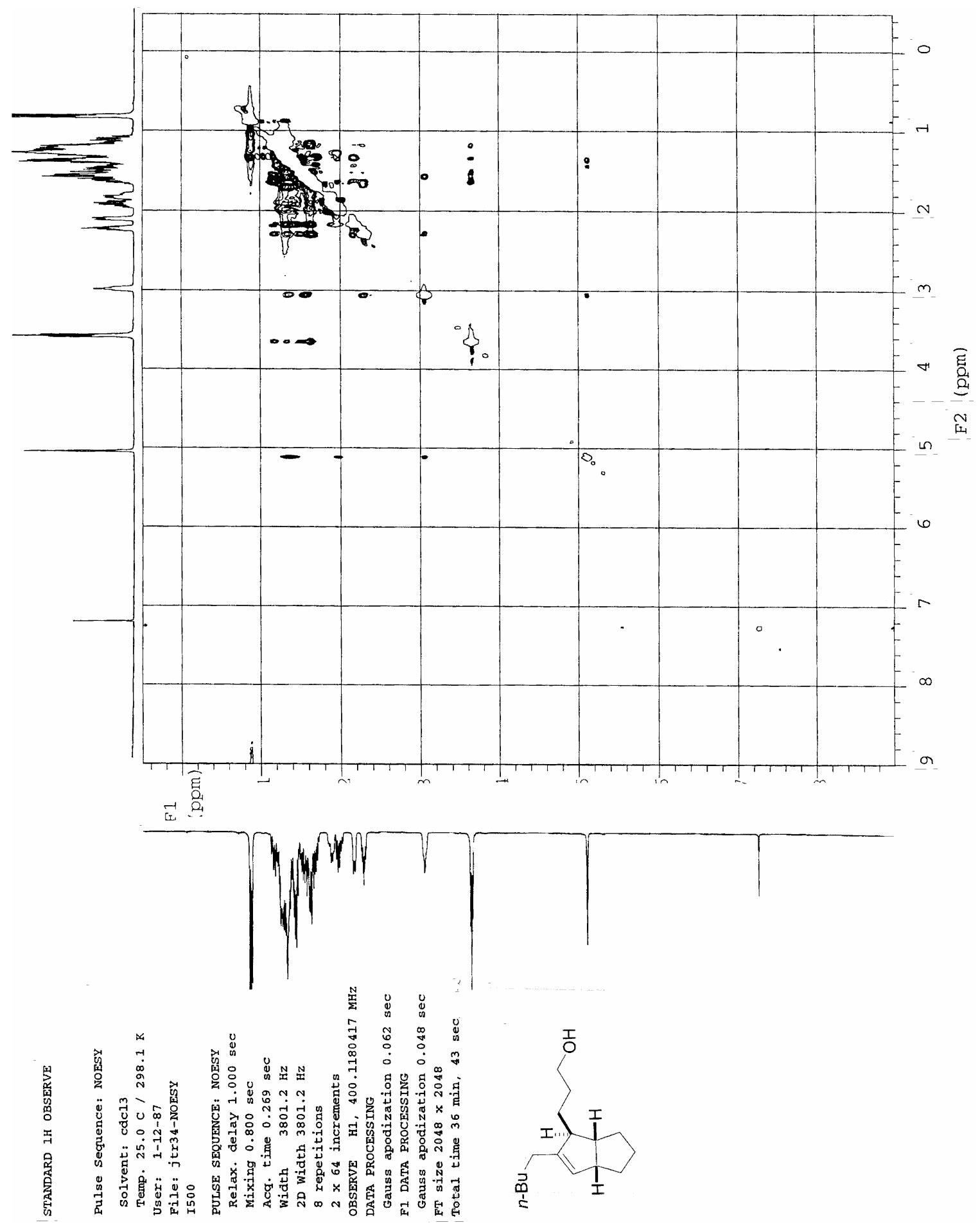

